\title{
On a New Epidemic Model with Asymptomatic and Dead-Infective Subpopulations with Feedback Controls Useful for Ebola Disease
}

\author{
M. De la Sen, ${ }^{1}$ A. Ibeas, ${ }^{2}$ S. Alonso-Quesada, ${ }^{1}$ and R. Nistal ${ }^{1}$ \\ ${ }^{1}$ Institute of Research and Development of Processes IIDP, University of the Basque Country, Campus of Leioa, P.O. Box 48940, \\ Leioa, Bizkaia, Spain \\ ${ }^{2}$ Department of Telecommunications and Systems Engineering, Universitat Autònoma de Barcelona (UAB), 08193 Barcelona, Spain
}

Correspondence should be addressed to M. De la Sen; manuel.delasen@ehu.eus

Received 13 December 2016; Revised 9 January 2017; Accepted 15 January 2017; Published 19 February 2017

Academic Editor: Lu-Xing Yang

Copyright (C) 2017 M. De la Sen et al. This is an open access article distributed under the Creative Commons Attribution License, which permits unrestricted use, distribution, and reproduction in any medium, provided the original work is properly cited.

\begin{abstract}
This paper studies the nonnegativity and local and global stability properties of the solutions of a newly proposed SEIADR model which incorporates asymptomatic and dead-infective subpopulations into the standard SEIR model and, in parallel, it incorporates feedback vaccination plus a constant term on the susceptible and feedback antiviral treatment controls on the symptomatic infectious subpopulation. A third control action of impulsive type (or "culling") consists of the periodic retirement of all or a fraction of the lying corpses which can become infective in certain diseases, for instance, the Ebola infection. The three controls are allowed to be eventually time varying and contain a total of four design control gains. The local stability analysis around both the disease-free and endemic equilibrium points is performed by the investigation of the eigenvalues of the corresponding Jacobian matrices. The global stability is formally discussed by using tools of qualitative theory of differential equations by using GaussStokes and Bendixson theorems so that neither Lyapunov equation candidates nor the explicit solutions are used. It is proved that stability holds as a parallel property to positivity and that disease-free and the endemic equilibrium states cannot be simultaneously either stable or unstable. The periodic limit solution trajectories and equilibrium points are analyzed in a combined fashion in the sense that the endemic periodic solutions become, in particular, equilibrium points if the control gains converge to constant values and the control gain for culling the infective corpses is asymptotically zeroed.
\end{abstract}

\section{Introduction}

Relevant attention is being paid in the last two decades to the study of mathematical epidemic models which are modelled by integro-differential equations and/or difference equations. Those models describe the evolution of the various subpopulations considered as the disease under study progresses. Typically, the models have three essential subpopulations (namely, susceptible, infected, and recovered by immunity) whose dynamics are mutually coupled. There are different degrees of complexity in the statement of the models. The simplest ones have only "susceptible" $(S)$ and "infected" $(I)$ subpopulations and are referred to as SI-models. A second degree of complexity adds a third one said to be the "recovered by immunity" subpopulation and those models are said to be SIR-models. A further complexity degree splits the infected into two subpopulations (or compartments), namely, the so-called "infected" or "exposed" $(E)$ subpopulation (those having the disease but do not present yet external symptoms) and the "infectious" or "infective" subpopulation (those having external symptoms). The generic acronym used for this last category of models is SEIR, being referred to as SEIR epidemic models. General description of epidemic models and some mathematical analysis on them is given in some classical books. See, for instance, [1-3] and for more recent models, see, for instance, [4-11] and references therein. The positivity of the solution is investigated in a number of works. See, for instance, $[6-9,12]$ and some references therein. The use of nonlinear incidence rates in the models is also investigated in a number of papers. See, for instance, [13-15]. The presence of perturbations is also investigated in many models. See, for instance, $[9,15-17]$ 
to give some of them. Also, certain robustness studies of stability and positivity under deviations of the equilibrium points due to Wiener noise are performed in [9]. The stability properties and the convergence of the solutions to equilibrium states are a major analysis tool in most of the works. In particular, the asymptotic solution behaviors including associated diffusion effects have been provided in $[18,19]$ and some references therein. The use of vaccination rules to improve the infection behavior has been also proposed in the literature. See, for instance, $[6-8,11,20-23]$ and references therein. In particular, two control actions are proposed in [20], namely, a vaccination action of the susceptible and a therapeutic treatment of the infectious subpopulation with constant and nonconstant controls and impulsive controls are proposed in $[22,23]$. The stability and optimal control under a subpopulation of infective in treatment with vaccination is investigated in [24] and a model with delay, latent period and saturation incidence rate and impulsive vaccination is proposed and discussed in [25].

On the other hand, it turns out as known due to medical experience that there are individuals who are infective but do not have significant external symptoms, that is, the socalled the "asymptomatic" $(A)$ subpopulation, [26]. This occurs even in the common known influenza disease. If such an asymptomatic subpopulation is considered in the model, then it turns out that the exposed subpopulation have different transitions to the symptomatic infectious subpopulation and to the asymptomatic ones so that a part of the exposed become subpopulation asymptomatic infectious after a certain time while others become symptomatic infectious. Finally, it is well known that in the case of Ebola disease, the lying dead corpses are infective $[27,28]$ which causes serious sanitary problems in third world tropical countries with low or scarce sanitary means when an Ebola disease spreads thoroughly specially when it is transmitted from rural areas to high populated urban ones. The dead corpses can be considered in the model as a new subpopulation " $D$."

The paper is organized as follows. Section 2 defines the SEIADR model with the six subpopulations $(S, E, I, A, D, R)$ under controls in terms of vaccination control on the susceptible and antiviral treatment on the symptomatic infectious subpopulation. The vaccination control possesses feedbackindependent (which can be constant, in particular) and feedback linear terms while the antiviral treatment control is implemented via proportional gain acting on the symptomatic infectious population. There is also a third control which consists of an impulsive control action of retirement of corpses to reduce the risks of dead-contagion to the living uninfected population. The three mentioned controls have feedback information taken on line from their respective subpopulations. The nonconstant control terms are based on feedback information of the respective subpopulations. Section 2 also discusses later on some nonnegativity and stability properties of the model, under the various controls, in a linked way in the sense that the nonnegativity of the subpopulations, under nonzero initial conditions, and the boundedness of the total population both together guarantee the boundedness of all the subpopulations for all time as a result. Section 3 deals with the disease-free and endemic equilibrium points and the periodic limit solutions of the controlled epidemic model as well as the associated local stability properties. The dependence of the resulting diseasefree and endemic equilibrium states is seen to be dependent on the limiting vaccination control gains. On the other hand, the global stability is also investigated by using qualitative theory of stability of differential equations by using GaussStokes and Bendixson theorems while neither Lyapunov functions nor the explicit solutions of the differential model are invoked at this stage. Finally, some numerical examples are given in Section 4 with attention to oscillatory behaviors under periodic culling action of dead infectious corpses and some conclusions end the paper.

\subsection{Notation}

$\mathbf{R}_{+}=\{r \in \mathbf{R}: r>0\} ; \mathbf{R}_{0+}=\{r \in \mathbf{R}: r \geq 0\}$,

C is the complex plane,

$\vee$ and $\wedge$ stand, respectively, for logic "or" and "and,"

$C^{0}$ and $P C^{0}$ are, respectively, the sets of continuous and piecewise-continuous functions of domain $I$ and image $X$. The functions $f: I \rightarrow X$ in those sets are denoted, respectively, by $f \in C^{0}(I, X)$ and $f \in$ $P C^{0}(I, X)$,

$\operatorname{card}(A)$ denotes the cardinal of the set $A$,

$\operatorname{card}(A)=\aleph_{0}$ indicates that the cardinal of a denumerable set $A$ is infinite as opposed to $\operatorname{card}(A)=\infty$, denoting the infinity cardinal of a nondenumerable set $A$,

$\mathbf{I}_{n}$ is $n$th identity matrix,

$\delta(t)$ denotes the Dirac distribution at $t=0$,

$\bar{m}=\{1,2, \ldots, m\}$.

\section{The SEIADR Epidemic Model: Some Results on Nonnegativity, Stability, and Equilibrium Solution Trajectories}

The proposed SEIADR model is an extended SEIR model with the following characteristics and novelties:

(a) Apart from the classical subpopulations of "susceptible" $(S)$, "exposed" who are infected but not yet infective $(E)$, "symptomatic infectious" $(I)$, and "recovered" $(R)$ subpopulation, it has two extra additional subpopulations, namely, "asymptomatic infectious" $(A)$ and "dead-infective" $(D)$. The so-called asymptomatic are a group of infective individuals (which are modelled as a distinct group of the $I$-infective subpopulation), characterized by small or null level of infection, with acquired immunity, but who can transmit the infective disease to others. The so-called dead-infective subpopulation are dead individuals (spread corpses in the distribution disease habitat) which transmit the illness because of lack of good sanitary performance or practice in certain infective 
illnesses (e.g., the Ebola disease) as it is a common situation in some third world countries with scarce technical and economic means.

(b) It incorporates three combined control actions which can be of a feedback nature as follows: (1) the standard vaccination control $V$ of the susceptible which consists of two terms, one of them being a nonfeedback gain and another feedback term with a gain being proportional to the susceptible, (2) the antiviral treatment $\xi$ of the infective subpopulation with a proportional gain on the symptomatic infectious subpopulation, and (3) the dead-infective culling which has a feedback impulsive nature modulated by a control gain in the sense that it is not applied at all time but at certain periods where either voluntary or civil-servant staff can become involved on this duty. The three controls contain together four, eventually time varying, design control gains which is a novel contribution of the paper related to the background literature while another novelty is the global stability analysis outlined from qualitative theory of differential equations.

It has been pointed out that the coexistence of an asymptomatic infectious subpopulation, often known in some wellknown diseases as influenza, and a dead-infective subpopulation (e.g., in the case of the Ebola) can occur. See, for instance, a related UK medical report [29] and see also [27]. Recent work on the incorporation of infective corpses and asymptomatic infectious type as new subpopulation is discussed, for instance, in $[26,28]$. The epidemic SEIADR model with vaccination and antiviral treatment together with infective corpses culling is as follows:

$$
\begin{aligned}
& \dot{S}(t)=b_{1}-\left(b_{2}+\beta I(t)+\beta_{A} A(t)+\beta_{D} D(t)\right) S(t) \\
& +\eta R(t)-V(t), \\
& \dot{E}(t)=-\left(b_{2}+\gamma\right) E(t) \\
& \quad+\left(\beta I(t)+\beta_{A} A(t)+\beta_{D} D(t)\right) S(t), \\
& \dot{I}(t)=-\left(b_{2}+\alpha+\tau_{0}\right) I(t)+\gamma p E(t)-\xi(t), \\
& \dot{A}(t)=-\left(b_{2}+\tau_{0}\right) A(t)+\gamma(1-p) E(t), \\
& \dot{D}(t)=-\mu D(t)+b_{2}(I(t)+A(t))+\alpha I(t) \\
& \quad-\rho_{D}(t) D(t) \sum_{t_{i} \in \operatorname{Imp} D} \delta\left(t-t_{i}\right), \\
& \dot{R}(t)=-\left(b_{2}+\eta\right) R(t)+\tau_{0}(I(t)+A(t))+\xi(t) \\
& \quad+V(t), \\
& V(t)=V_{0}(t)+K_{V}(t) S(t), \\
& \xi(t)=K_{\xi}(t) I(t) ;
\end{aligned}
$$

with initial conditions satisfying $\min (S(0), E(0), I(0), A(0)$, $D(0), R(0)) \geq 0$, where $\operatorname{Imp} D=\left\{t \in \mathbf{R}_{0+}: D(t) \neq D\left(t^{-}\right)\right\}=$ $\bigcup_{t \in \mathbf{R}_{0+}} \operatorname{Imp} D(t)$ is the total set of impulsive ("culling") time instants for removal of infective corpses (note that the notation for $f\left(t^{+}\right)$is simplified to $\left.f(t)\right)$. The vaccination $V(t)$ and (7) consist of feedback-independent term, which can be constant, plus a linear feedback term injected on the susceptible subpopulation while the antiviral action is a linear feedback control applied to the symptomatic infectious subpopulation. Besides,

$$
\begin{aligned}
\operatorname{Imp} D\left(t^{-}\right) & =\{\sigma \in \operatorname{Imp} D: \sigma<t\}, \\
\operatorname{Imp} D(t) & =\{\sigma \in \operatorname{Imp} D: \sigma \leq t\}=\operatorname{Imp} D\left(t^{-}\right) \\
& \text {if } t \notin \operatorname{Imp} D, \\
\operatorname{Imp} D(t) & =\{\sigma \in \operatorname{Imp} D: \sigma \leq t\}=\operatorname{Imp} D\left(t^{-}\right) \cup\{t\} \\
& \text { if } t \in \operatorname{Imp} D
\end{aligned}
$$

and the (nonnegative) parameters and controls are the following:

$b_{1}$ is the recruitment rate.

$b_{2}$ is the natural average death rate.

$\beta, \beta_{A}, \beta_{D}$ are the various disease transmission coefficients to the susceptible from the respective symptomatic infectious, asymptomatic, and infective corpses subpopulations.

$\eta$ is a parameter such that $1 / \eta$ is the average duration of the immunity period reflecting a transition from the recovered to the susceptible.

$\gamma$ is the transition rate from the exposed to all (i.e., both symptomatic and asymptomatic) infectious subpopulation.

$\alpha$ is the average extra mortality associated with the symptomatic infectious subpopulation.

$\tau_{0}$ is the natural immune response rate for the whole infectious subpopulation (i.e., $A+I$ ), respectively; $p$ is the fraction of the exposed which become symptomatic infectious subpopulation.

$1-p$ is the fraction of the exposed which becomes asymptomatic infectious subpopulation.

$1 / \mu$ is the average period of infectiousness after death.

$V(t)$ and $\xi(t)$ are, respectively, the vaccination and antiviral treatment controls and $\rho_{D}\left(t_{i}\right) D\left(t_{i}\right)$ is the impulsive action of removal of corpses (or "culling") for all $t_{i} \in \operatorname{Imp} D$ with some piecewise continuous $\rho_{D}(t) \in[0,1]$. The controls can be of different types including constant and feedback actions. It turns out that a well-posed epidemic model has to be positive and with bounded solutions to be useful for potential applications. The subsequent results are, respectively, related to the nonnegativity under nonnegative initial conditions and some smoothness conditions on the controls and boundedness of the solutions of the 
model. Note that the positivity of the trajectory solutions as well as that of the equilibrium solutions is a crucial "a priori" basic requirement for model validation in many different biological problems. See, for instance, [6-9, 12, 18, 30-32].

Theorem 1. The solutions of the SEIADR model (1) to (8) are uniquely defined and if $\min (S(0), E(0), I(0), A(0), R(0)$, $D(0)) \geq 0, V_{0}(t) \in\left[0, b_{1}+\eta R(t)\right], \rho_{D}, V, K_{V}, K_{\xi} \in P C^{0}\left(\mathbf{R}_{0+}\right.$, $\left.\mathbf{R}_{0+}\right)$ and $\rho_{D}: \mathbf{R}_{0+} \rightarrow[0,1]$, then such solutions are, furthermore, nonnegative for any given nonnegative initial conditions defined by:

$$
\begin{aligned}
& S(t)=e^{-\left(b_{2} t+\int_{0}^{t}\left(K_{V}(\sigma)+\beta I(\sigma)+\beta_{A} A(\sigma)+\beta_{D} D(\sigma)\right) d \sigma\right)} \times(S(0) \\
& +\int_{0}^{t} e^{\int_{0}^{\sigma}\left(b_{2}+K_{V}(\theta)+\beta I(\theta)+\beta_{A} A(\theta)+\beta_{D} D(\theta)\right) d \theta}\left(b_{1}+\eta R(\sigma)\right. \\
& \left.\left.-V_{0}(\sigma)\right) d \sigma\right) ; \quad \forall t \in \mathbf{R}_{0+} \\
& E(t)=e^{-\left(b_{2}+\gamma\right) t}\left(E(0)+\int_{0}^{t} e^{\left(b_{2}+\gamma\right) \sigma}\left(\beta I(\sigma)+\beta_{A} A(\sigma)\right.\right. \\
& \left.\left.+\beta_{D} D(\sigma)\right) S(\sigma) d \sigma\right) ; \quad \forall t \in \mathbf{R}_{0+}, \\
& I(t)=e^{-\left(\left(b_{2}+\alpha+\tau_{0}\right) t+\int_{0}^{t} K_{\xi}(\sigma) d \sigma\right)}(I(0) \\
& \left.+\gamma p \int_{0}^{t} e^{\int_{0}^{\sigma}\left(b_{2}+\alpha+\tau_{0}+K_{\xi}(\theta)\right) d \theta} E(\sigma) d \sigma\right) ; \quad \forall t \in \mathbf{R}_{0+}, \\
& A(t)=e^{-\left(b_{2}+\tau_{0}\right) t}(A(0)+\gamma(1-p) \\
& \left.\cdot \int_{0}^{t} e^{\left(b_{2}+\tau_{0}\right) \sigma} E(\sigma) d \sigma\right) ; \quad \forall t \in \mathbf{R}_{0+}, \\
& R(t)=e^{-\left(b_{2}+\eta\right) t}\left(R(0)+\int_{0}^{t} e^{\left(b_{2}+\eta\right) \sigma}\left(\tau_{0}(I(\sigma)+A(\sigma))\right.\right. \\
& \left.\left.+K_{\xi}(\sigma) I(\sigma)+V_{0}(\sigma)+K_{V}(\sigma) S(\sigma)\right) d \sigma\right) ; \\
& \forall t \in \mathbf{R}_{0+} \text {, } \\
& D(t)=e^{-\mu\left(t-t_{i}\right)}\left(D\left(t_{i}\right)+\int_{t_{i}}^{t} e^{\mu\left(\sigma-t_{i}\right)}\left[\left(b_{2}+\alpha\right) I(\sigma)\right.\right. \\
& \left.\left.+b_{2} A(\sigma)\right] d \sigma\right)
\end{aligned}
$$$$
\forall t \in\left[t_{i}, t_{i+1}\right), \forall t_{i} \in \operatorname{Imp} D
$$

with

$$
\begin{aligned}
& D\left(t_{i+1}^{-}\right)=e^{-\mu T_{i}}\left(D\left(t_{i}\right)\right. \\
& \left.\quad+\int_{t_{i}}^{t_{i+1}} e^{\mu\left(\sigma-t_{i}\right)}\left[\left(b_{2}+\alpha\right) I(\sigma)+b_{2} A(\sigma)\right] d \sigma\right)
\end{aligned}
$$

while

$$
\begin{gathered}
D\left(t_{i+1}\right)=D\left(t_{i+1}^{-}\right)-\int_{t_{i+1}^{-}}^{t_{i+1}} \rho_{D}(\sigma) D(\sigma) \delta\left(\sigma-t_{i+1}\right) d \sigma \\
=\left(1-\rho_{D}\left(t_{i+1}\right)\right) D\left(t_{i+1}^{-}\right)=\left(1-\rho_{D}\left(t_{i+1}\right)\right) \\
\quad \cdot e^{-\mu T_{i}}\left(D\left(t_{i}\right)\right. \\
\left.\quad+\int_{t_{i}}^{t_{i+1}} e^{\mu\left(\sigma-t_{i}\right)}\left[\left(b_{2}+\alpha\right) I(\sigma)+b_{2} A(\sigma)\right] d \sigma\right),
\end{gathered}
$$

where $T_{i}=t_{i+1}-t_{i} ; \forall t_{i} \in \operatorname{ImpD}$. Furthermore, $S, E, I, A, R \in$ $C^{0}\left(\mathbf{R}_{0+}, \mathbf{R}_{0+}\right)$ are everywhere differentiable in $\mathbf{R}_{0+}$ and $D \in$ $P C^{0}\left(\mathbf{R}_{0+}, \mathbf{R}_{0+}\right)$ and it is time-differentiable in $\bigcup_{t_{i} \in \operatorname{Imp} D}\left(t_{i}, t_{i+1}\right)$.

Proof. The replacements of (7) into (1) and (8) into (3) yield

$$
\begin{aligned}
& \dot{S}(t)=b_{1} \\
&-\left(b_{2}+K_{V}(t)+\beta I(t)+\beta_{A} A(t)+\beta_{D} D(t)\right) S(t) \\
&+\eta R(t)-V_{0}(t), \\
& \dot{I}(t)=-\left(b_{2}+\alpha+\tau_{0}+K_{\xi}(t)\right) I(t)+\gamma p E(t) ; \\
& \forall t \in \mathbf{R}_{0+} .
\end{aligned}
$$

The solutions of (19), (2), (20), and (4)-(6) follow via direct calculus and are unique and nonnegative resulting in (11)(18) for any given set of nonnegative initial conditions. Also, $S, E, I, A, R \in C^{0}\left(\mathbf{R}_{0+}, \mathbf{R}_{0+}\right)$ since their first respective time derivatives exist everywhere in $\mathbf{R}_{0+}$ from (1)-(4) and (6). Furthermore, note from (5) and the fact that its impulsive ("culling") control $\rho_{D}: \mathbf{R}_{0+} \rightarrow[0,1]$ yields a unique piecewise solution $D \in P C^{0}\left(\mathbf{R}_{0+}, \mathbf{R}_{0+}\right)$ for each given $D(0)$.

The boundedness of all the subpopulations for all time and the asymptotic infection removal under a feedback, in general, time-varying linear antiviral control law, is addressed by the subsequent result.

Theorem 2. The following properties hold under the assumptions of Theorem 1:

(i) $\limsup \sup _{t \rightarrow \infty} I(t) \leq b_{1} / \alpha, \sup _{t \in \mathbf{R}_{0+}} I(t)<+\infty$, $\sup _{t \in \mathbf{R}_{0+}}$ $N(t) \leq N(0)+b_{1} / b_{2}<+\infty ; \forall t \in \mathbf{R}_{0+}$ where $N(t)=$ $S(t)+E(t)+I(t)+A(t)+R(t) ; \forall t \in \mathbf{R}_{0+}$ is the total alive population, and

$$
\begin{aligned}
& \max \left(\sup _{t \in \mathbf{R}_{0+}} S(t), \sup _{t \in \mathbf{R}_{0+}} E(t), \sup _{t \in \mathbf{R}_{0+}} I(t), \sup _{t \in \mathbf{R}_{0+}} A(t),\right. \\
& \left.\sup _{t \in \mathbf{R}_{0+}} D(t), \sup _{t \in \mathbf{R}_{0+}} R(t)\right) \leq \sup _{t \in \mathbf{R}_{0+}} \bar{N}(t) \\
& \quad \leq \max \left(\sup _{t \in \mathbf{R}_{0+}} N(t), \sup _{t \in \mathbf{R}_{0+}} D(t)\right)<+\infty,
\end{aligned}
$$


(ii) for any $t \in \mathbf{R}_{0+}$, assume that $K_{\xi}(t)=0$ if $I(t)=0$, and the antiviral control gain is chosen to be

$$
\begin{aligned}
& K_{\xi}(t)=\frac{\xi(t)}{I(t)}=\frac{1}{I(t)}\left[\left(\alpha+\tau_{0}\right) E(t)+\alpha A(t)\right. \\
& \left.\quad+\left(\beta_{A} A(t)+\beta_{D} D(t)\right) S(t)\right]+\beta S(t) \quad \text { if } I(t) \neq 0 .
\end{aligned}
$$

Then, $K_{\xi}(t)=O(I(t))$, implying also that $\sup _{t \in \mathbf{R}_{0+}}$ $K_{\xi}(t)<+\infty$, and the following limits exist:

$$
\begin{aligned}
& \lim _{t \rightarrow \infty}(E(t)+I(t)+A(t)+D(t))=0, \\
& \lim _{t \rightarrow \infty}(S(t)+R(t))=\lim _{t \rightarrow \infty} \bar{N}(t)=\lim _{t \rightarrow \infty} N(t)=\frac{b_{1}}{b_{2}},
\end{aligned}
$$

where $\bar{N}(t)=N(t)+D(t) ; \forall t \in \mathbf{R}_{0+}$ is the total population including infective corpses.

(iii) If, furthermore, $V_{0}(t)$ satisfies the most stringent constraint $\limsup _{t \rightarrow \infty}\left(V_{0}(t)-b_{1}-\eta R(t)+\varepsilon_{V}\right) \leq$ 0 for any fixed $\varepsilon_{V}\left(\leq b_{1}-\eta R(t)\right) \in \mathbf{R}_{+}$, then $\min \left(\lim \inf _{t \rightarrow \infty} S(t), \liminf _{t \rightarrow \infty} R(t)\right)>0$.

Proof. Assume that $\limsup _{t \rightarrow \infty} I(t)>b_{1} / \alpha$ and proceed by contradiction. By summing up (1) to (4) and adding (6), one gets $\dot{N}(t)=-b_{2} N(t)+b_{1}-\alpha I(t) ; \forall t \in \mathbf{R}_{0+}$ which concludes that

$$
\limsup _{t \rightarrow \infty}\left(\int_{0}^{t} e^{-b_{2}(t-\sigma)}\left(\alpha I(\sigma)-b_{1}\right) d \sigma+N(t)\right)=0 .
$$

Since $\lim \sup _{t \rightarrow \infty} I(t)>b_{1} / \alpha$ and $N \in C^{0}\left(\mathbf{R}_{0+}, \mathbf{R}_{0+}\right)$, which is derived from the result of Theorem 1, it follows a contradiction to (25) since lim $\sup _{t \rightarrow \infty}\left(\int_{0}^{t} e^{-b_{2}(t-\sigma)}\left(\alpha I(\sigma)-b_{1}\right) d \sigma+\right.$ $N(t))>0$. Therefore, $\lim \sup _{t \rightarrow \infty} I(t) \leq b_{1} / \alpha<+\infty$. Also, the boundedness of $N(t)$ follows directly since $I(t) \geq 0 ; \forall t \in \mathbf{R}_{0+}$ from the standard comparison theorem for $\dot{N}(t) \leq \dot{N}_{0}(t)=$ $-b_{2} N_{0}(t)+b_{1}$ leading to $N(t) \leq e^{-b_{2} t} N(0)+\left(1-e^{-b_{2} t}\right)\left(b_{1} / b_{2}\right) \leq$ $N(0)+b_{1} / b_{2}<+\infty ; \forall t \in \mathbf{R}_{0+}$ provided that $N_{0}(0)=N(0)$ and $\lim \sup _{t \rightarrow \infty} N(t)=b_{1} / b_{2}$. From Theorem 1 , all the subpopulations are nonnegative for all time for any given nonnegative initial conditions. Since the model is nonnegative for all time then all the living subpopulations are bounded for all time since $N(t)<+\infty$. From (17)-(18) the lying corpses subpopulation is nonnegative and bounded for all time since both the symptomatic and asymptomatic infectious subpopulations are bounded for all time. As a result, the total population is also bounded for all time as they are all the subpopulations. Property (i) is proved. To prove Property (ii), one gets from (2), (3), and (4) under the given antiviral treatment control law that

$$
\begin{aligned}
\dot{E}(t) & +\dot{I}(t)+\dot{A}(t) \\
= & -b_{2}(E(t)+I(t)+A(t)) \\
& +\left(\beta I(t)+\beta_{A} A(t)+\beta_{D} D(t)\right) S(t) \\
& -\left(\alpha+\tau_{0}\right) I(t)-\xi(t)-\tau_{0} A(t)
\end{aligned}
$$

$$
\begin{aligned}
= & -\left(b_{2}+\tau_{0}+\alpha\right)(E(t)+I(t)+A(t)) \\
& +\left(\beta I(t)+\beta_{A} A(t)+\beta_{D} D(t)\right) S(t) \\
& +\left(\alpha+\tau_{0}\right) E(t)+\alpha A(t)-K_{\xi}(t) I(t) \\
= & -\left(b_{2}+\tau_{0}+\alpha\right)(E(t)+I(t)+A(t)) ;
\end{aligned}
$$$$
\forall t \in \mathbf{R}_{0+}
$$

so that it exists the limit $\lim _{t \rightarrow \infty}(E(t)+I(t)+A(t))=$ $e^{-\left(b_{2}+\tau_{0}+\alpha\right) t}(E(0)+I(0)+A(0))=0$. Thus, $\lim _{t \rightarrow \infty} E(t)=$ $\lim _{t \rightarrow \infty} I(t)=\lim _{t \rightarrow \infty} A(t)=0$ since the three subpopulations are nonnegative for all time under any given nonnegative initial conditions. This also implies as a result that $\lim _{t \rightarrow \infty}(S(t)+R(t))=\lim _{t \rightarrow \infty} \bar{N}(t)=\lim _{t \rightarrow \infty} N(t)=$ $b_{1} / b_{2}$ since from $(16)-(18), \lim _{t \rightarrow \infty} D(t)=0$. It remains to prove that $K_{\xi}(t)=O(I(t))=O(\max (I(t), S(t))<+\infty)$. First, note that $I(t)$ is uniformly bounded since it is nonnegative and the total population is uniformly bounded. Thus, to prove that $K_{\xi}(t)=O(I(t))=O(I(t), S(t))$, it suffices to prove, in view of (23), that $I \leq \max (o(E), o(A), o(D))$. Since $\lim _{t \rightarrow \infty}(E(t)+I(t)+A(t))=0$, then $\lim _{t \rightarrow \infty}(E(t)+A(t))=0$. On the other hand, note from (13) that $I(t) \rightarrow 0$ as $t \rightarrow t_{1}$ for any $t_{1} \in \mathbf{R}_{0+}$ implies $\int_{0}^{t_{1}} e^{-\left(\left(b_{2}+\alpha+\tau_{0}\right)\left(t_{1}-\sigma\right)+\int_{\sigma}^{t_{1}} K_{\xi}(\sigma) d \sigma\right)} E(\sigma) d \sigma \rightarrow$ 0 and $E\left(t_{1}\right) \rightarrow 0$. If, in addition $I(0)>0$ then $t_{1} \rightarrow \infty$. On the other hand, from (12) if $E(t) \rightarrow 0$ as $t \rightarrow \infty$, then $I(t), A(t), D(t) \rightarrow 0$ as $t \rightarrow \infty$. Thus, $E(t) / I(t)$ and $A(t) / I(t)$ cannot diverge as $t \rightarrow \infty$ if $E(t) \rightarrow 0$ as $t \rightarrow \infty$. Thus, if $I(t) \rightarrow 0$ then $E(t), A(t), D(t) \rightarrow 0$ and if $E(t) \rightarrow 0$ or $A(t) \rightarrow 0$ (see also (14)), then $I(t) \rightarrow 0$. Then, $K_{\xi}(t)=$ $O(I(t))=O(I(t), S(t))$. Property (ii) has been proved. On the other hand, if $\liminf _{t \rightarrow \infty}\left(b_{1}-\varepsilon_{V}+\eta R(t)-V_{0}(t)\right) \geq 0$ then $\liminf _{t \rightarrow \infty} S(t)>0$ from (11) which leads to $\liminf _{t \rightarrow \infty} R(t)>$ 0 from (15). Hence, Property (iii) is proved.

Remark 3. Note that the condition $\lim \inf _{t \rightarrow \infty}\left(b_{1}-\varepsilon_{V}+\eta R(t)-\right.$ $\left.V_{0}(t)\right) \geq 0$ for $\varepsilon_{V}=0$ of Theorem 2(iii) is guaranteed if $V_{0}(t) \epsilon$ $\left[0, b_{1}\right) ; \forall t \in \mathbf{R}_{0+}$.

\section{Disease-Free and Endemic Equilibrium Points, Limit Periodic Equilibrium Trajectories, and Local and Global Stability}

Define the linearized error of the trajectory solution with respect to any equilibrium $x^{*}$ by

$$
\tilde{x}(t)=x(t)-x^{*}(t) ; \quad \forall t \in \mathbf{R}_{0+} \backslash \operatorname{Imp} D,
$$

where $x(t)$ is the linearized state-trajectory solution in $\mathbf{R}_{0+}^{6}$ whose six components are defined by $S(t), E(t), I(t)$, $A(t), D(t)$, and $R(t)$ in this order. In particular, $x_{\mathrm{df}}^{*}(t)=$ $x_{\mathrm{df}}^{*}=\left(S_{\mathrm{df}}^{*}, 0,0,0,0, R_{\mathrm{df}}^{*}\right)^{T}$ for any $t \in \mathbf{R}_{0+}$ is the diseasefree equilibrium solution, which is an equilibrium point, and $x_{\text {end }}^{*}(t)=\left(S_{\text {end }}^{*}(t), E_{\text {end }}^{*}(t), I_{\text {end }}^{*}(t), A_{\text {end }}^{*}(t), D_{\text {end }}^{*}(t), R_{\text {end }}^{*}(t)\right)^{T}$ for any $t \in\left[0, T_{D}^{*}\right]$ is an equilibrium periodic trajectory of period $T_{D}^{*}$ if $\rho_{D}(t) \rightarrow \rho_{D}^{*} \in(0,1)$ and $\left(t_{i+1}-t_{i}\right) \rightarrow T_{D}^{*}(>0)$ as $t_{i}(\in \operatorname{Imp} D) \rightarrow \infty$. If $\rho_{D}^{*}=0$ or $\operatorname{card} \operatorname{Imp} D<\chi_{0}$ 
(i.e., the cardinal of impulsive time instants is numerable finite), then $x_{\text {end }}^{*}(t)=x_{\text {end }}^{*} ; \forall t \in \mathbf{R}_{0+}$ (i.e., the limit periodic endemic solution is just an endemic equilibrium point). The following result holds and is concerned with the eventually periodic asymptotic behavior of the dead-infective lying corpses subpopulation under constant limiting values of the culling removal fraction and culling period. It is also obtained the intuitively obvious result that if all the lying infective corpses are removed by the culling control then the dead corpses infective subpopulation is asymptotically zeroed at the culling time instants.

Theorem 4. The following properties hold:

(i) Assume that $\left(t_{i+1}-t_{i}\right) \rightarrow T_{D}^{*}(>0), V_{0}(t)=V_{0} ; \forall t \in$ $\mathbf{R}_{0+}$, and $\rho_{D}\left(t_{i}\right) \rightarrow \rho_{D}^{*}(\in[0,1])$ as $t_{i}(\in \operatorname{Imp} D) \rightarrow \infty$. Then, a periodic limit solution of period $T_{D}^{*}$ of the form

$$
\begin{aligned}
& \lim _{n \rightarrow \infty} D\left(n T_{D}^{*}+\theta\right)=D^{*}\left(T_{D}^{*}+\theta\right) \\
& \quad=\frac{e^{-\mu \theta}}{\mu}\left[\left(b_{2}+\alpha\right) I_{a v}^{*}+b_{2} A_{a v}^{*}\right] \\
& . {\left[\frac{\left(1-\rho_{D}^{*}\right)\left(1-e^{-\mu \theta}\right)}{\left(1-\left(1-\rho_{D}^{*}\right) e^{-\mu \theta}\right)}-1+e^{\mu \theta}\right] ; \quad \forall \theta \in\left[0, T_{D}^{*}\right] }
\end{aligned}
$$

exists for the dead-infective corpses subpopulation, where the subscript "av" stands for a mean value of the corresponding subpopulation on the period $\left[0, T_{D}^{*}\right)$ with existing right and left limits

$$
\begin{aligned}
D^{*}\left(T_{D}^{*}+\theta\right) & =\lim _{n \rightarrow \infty} D\left(n T_{D}^{*}+\theta\right)=\lim _{t_{i} \rightarrow \infty} D\left(t_{i}+\theta\right) ; \\
\forall \theta \in\left[0, T_{D}^{*}\right), & \\
D^{*}\left(T_{D}^{*-}\right)= & D\left(0^{-}\right)=\lim _{\theta \rightarrow 0^{-} n \rightarrow \infty} \lim _{n \rightarrow \infty} D\left(n T_{D}^{*}+\theta\right) \\
= & \lim _{\theta \rightarrow 0^{-}} \lim _{t_{i} \rightarrow \infty} D\left(t_{i}-\theta\right)
\end{aligned}
$$

possessing eventual discontinuities $D^{*}\left(T_{D}^{*}\right) \neq D^{*}\left(T_{D}^{*-}\right)$ which satisfy

$D^{*}\left(T_{D}^{*}\right)=\left(1-\rho_{D}^{*}\right) D^{*}\left(T_{D}^{*-}\right) ;$

$D^{*}\left(T_{D}^{*-}\right)$

$$
=\frac{1-e^{-\mu T_{D}^{*}}}{\mu\left(1-\left(1-\rho_{D}^{*}\right) e^{\left.-\mu T_{D}^{*}\right)}\right.}\left[\left(b_{2}+\alpha\right) I_{a v}^{*}+b_{2} A_{a v}^{*}\right] .
$$

(ii) If $T_{D}^{*}=+\infty$, or if $\operatorname{Imp} D$ has a finite cardinal, then

$$
\begin{aligned}
D^{*}\left(T_{D}^{*-}\right) & =\frac{1}{\mu}\left[\left(b_{2}+\alpha\right) I_{a v}^{*}+b_{2} A_{a v}^{*}\right] ; \\
D^{*}\left(T_{D}^{*}\right) & =\frac{1-\rho_{D}^{*}}{\mu}\left[\left(b_{2}+\alpha\right) I_{a v}^{*}+b_{2} A_{a v}^{*}\right] .
\end{aligned}
$$

If, furthermore $\rho_{D}^{*}=1$, then $D^{*}\left(T_{D}^{*}\right)=0$.

For the disease-free equilibrium, $D_{d f}^{*}\left(T_{D}^{*}\right)=D_{d f}^{*}$ $\left(T_{D}^{*-}\right)=0$ irrespective of $T_{D}^{*}$ and $\rho_{D}^{*}$.
If, furthermore $\rho_{D}^{*}=0$, then the endemic equilibrium periodic solution is an endemic equilibrium point $D_{\text {end }}^{*}=\left(\left(b_{2}+\alpha\right) I_{\text {end }}^{*}+b_{2} A_{\text {end }}^{*}\right) / \mu$.

(iii) The limit periodic solution $D^{*}\left(T_{D}^{*}+\theta\right)$ for $\theta \in\left[0, T_{D}^{*}\right)$ induces limit periodic oscillations of the susceptible and immune which obey the relationships:

$S^{*}(\theta)$

$$
=\frac{b_{1}-V_{0}+\eta R^{*}(\theta)}{b_{2}+K_{V}^{*}(\theta)+\beta I^{*}(\theta)+\beta_{A} A^{*}(\theta)+\beta_{D} D^{*}(\theta)},
$$

$$
R^{*}(\theta)=\frac{N_{R}^{*}(\theta)}{D_{R}^{*}(\theta)}
$$

where

$$
\begin{aligned}
& N_{R}^{*}(\theta)=\left(b_{2}+\beta I^{*}(\theta)+\beta_{A} A^{*}(\theta)+\beta_{D} D^{*}(\theta)\right) \\
& \cdot\left(\left(\tau_{0}+K_{\xi}^{*}(\theta)\right) I^{*}(\theta)+\tau_{0} A^{*}(\theta)+V_{0}^{*}(\theta)\right) \\
& +K_{V}^{*}(\theta)\left(\left(\tau_{0}+K_{\xi}^{*}(\theta)\right) I^{*}(\theta)+\tau_{0} A^{*}(\theta)+b_{1}\right), \\
& D_{R}^{*}(\theta)=\left(b_{2}+\eta\right) \\
& \quad \cdot\left(b_{2}+\beta I^{*}(\theta)+\beta_{A} A^{*}(\theta)+\beta_{D} D^{*}(\theta)\right) \\
& \quad+b_{2} K_{V}^{*}(\theta)
\end{aligned}
$$$$
\forall \theta \in\left[0, T_{D}^{*}\right]
$$

provided that $V_{0}\left(n T_{D}^{*}+\theta\right) \rightarrow V_{0}^{*}(\theta), K_{V}\left(n T_{D}^{*}+\theta\right) \rightarrow$ $K_{V}^{*}(\theta)$, and $K_{\xi}\left(n T_{D}^{*}+\theta\right) \rightarrow K_{\xi}^{*}(\theta)$ for any $\theta \in\left[0, T_{D}^{*}\right]$ as $n\left(\in \mathbf{Z}_{+}\right) \rightarrow \infty$. If $\rho_{D}^{*}=0, V_{0}^{*}(\theta)=V_{0}^{*}, K_{V}^{*}(\theta)=$ $K_{V}^{*}$, and $K_{\xi}(\theta)=K_{\xi}^{*} ; \forall \theta \in\left[0, T_{D}^{*}\right]$ then the endemic equilibrium solution is an endemic equilibrium point.

Proof. Note from (18) that if $\left(t_{i+1}-t_{i}\right) \rightarrow T_{D}^{*}$ and $\rho_{D}\left(t_{i}\right) \rightarrow$ $\rho_{D}^{*} \in[0,1]$ as $t_{i}(\in \operatorname{Imp} D) \rightarrow \infty$ then the right limits $D\left(T_{D}^{*}+\right.$ $\theta)=\lim _{n \rightarrow \infty} D\left(n T_{D}^{*}+\theta\right)=\lim _{t_{i} \rightarrow \infty} D\left(t_{i}+\theta\right)$ exist for $\theta \epsilon$ $\left[0, T_{D}^{*}\right)$ as well as the left limits

$D\left(T_{D}^{*-}\right)=\lim _{\theta \rightarrow 0^{-}} \lim _{n \rightarrow \infty} D\left(n T_{D}^{*}+\theta\right)=\lim _{\theta \rightarrow 0^{-}} \lim _{t_{i} \rightarrow \infty}$ $D\left(t_{i}-\theta\right)$ with eventual discontinuities $D\left(T_{D}^{*}\right) \neq D\left(T_{D}^{*-}\right)$. So, we have in the steady state

$$
\begin{aligned}
& D\left(t_{i+1}\right)=D\left(t_{i}\right)=D\left(T_{D}^{*}\right)=\left(1-\rho_{D}^{*}\right) D\left(t_{i+1}^{-}\right) \\
& \quad=\left(1-\rho_{D}^{*}\right) e^{-\mu T_{D}^{*}} D\left(T_{D}^{*}\right)+\left(1-\rho_{D}^{*}\right) \\
& \quad \cdot\left(\int_{0}^{T_{D}^{*}} e^{-\mu\left(T_{D}^{*}-\sigma\right)}\left[\left(b_{2}+\alpha\right) I^{*}(\sigma)+b_{2} A^{*}(\sigma)\right] d \sigma\right)
\end{aligned}
$$

so that, from the mean value theorem since the limit the periodic oscillation is bounded, there is a mean value of the symptomatic and asymptomatic infectious subpopulation such that

$$
\begin{gathered}
{\left[1-\left(1-\rho_{D}^{*}\right) e^{-\mu T_{D}^{*}}\right] D^{*}\left(T_{D}^{*}\right)=\left(1-\rho_{D}^{*}\right)} \\
\cdot \frac{1-e^{-\mu T_{D}^{*}}}{\mu}\left[\left(b_{2}+\alpha\right) I_{\mathrm{av}}^{*}+b_{2} A_{\mathrm{av}}^{*}\right],
\end{gathered}
$$




$$
\begin{gathered}
D^{*}\left(T_{D}^{*}+\theta\right)=e^{-\mu \theta} D^{*}\left(T_{D}^{*}\right)+\left[\left(b_{2}+\alpha\right) I_{\mathrm{av}}^{*}+b_{2} A_{\mathrm{av}}^{*}\right] \\
\cdot\left(\int_{0}^{\theta} e^{-\mu(\theta-\sigma)} d \sigma\right)=\frac{e^{-\mu \theta}}{\mu}\left[\left(b_{2}+\alpha\right) I_{\mathrm{av}}^{*}+b_{2} A_{\mathrm{av}}^{*}\right] \\
\cdot\left[\frac{\left(1-\rho_{D}^{*}\right)\left(1-e^{-\mu \theta}\right)}{\left(1-\left(1-\rho_{D}^{*}\right) e^{-\mu \theta}\right)}-1+e^{\mu \theta}\right] ;
\end{gathered}
$$

If $\rho_{D}^{*}=0$, one gets from (36) that

$$
\begin{gathered}
\lim _{t_{i}(\in \operatorname{Imp} D) \rightarrow \infty} D\left(t_{i}+\theta\right)=\left[\left(b_{2}+\alpha\right) I_{\mathrm{av}}^{*}+b_{2} A_{\mathrm{av}}^{*}\right] \\
\cdot \lim _{t_{i}(\in \operatorname{Imp} D) \rightarrow \infty}\left(\int_{t_{i}}^{t_{i}+T_{D}^{*}} e^{-\mu\left(T_{D}^{*}+\theta-\sigma\right)} d \sigma\right) \\
=D^{*}\left(T_{D}^{*}+\theta\right)=\frac{\left(b_{2}+\alpha\right) I_{\mathrm{av}}^{*}+b_{2} A_{\mathrm{av}}^{*}}{\mu}
\end{gathered}
$$

$$
\forall \theta \in\left[0, T_{D}^{*}\right)
$$

so that $D(t) \rightarrow 0$ as $t \rightarrow \infty$ if the disease-free equilibrium point is globally asymptotically attractive and $D(t) \rightarrow D_{\text {end }}^{*}=$ $\left(\left(b_{2}+\alpha\right) I_{\text {end }}^{*}+b_{2} A_{\text {end }}^{*}\right) / \mu$ if the endemic equilibrium state, which is an equilibrium point, is globally asymptotically attractive. The proofs of Properties (i)-(ii) are complete. To prove Property (iii), the inspection of (1) and (6) at any equilibrium yields that $S$ and $R$ have periodic oscillation if $D$ is periodic. So, we can get from (1) and (6) that if $V_{0}(\theta)=$ $V_{0}^{*}(\theta), K_{V}(\theta)=K_{V}^{*}(\theta)$, and $K_{\xi}(\theta)=K_{\xi}^{*}(\theta)$, for any $\theta \in$ $\left[0, T_{D}^{*}\right]$, the relations

$S^{*}(\theta)$

$$
\begin{aligned}
&=\frac{b_{1}-V_{0}^{*}(\theta)+\eta R^{*}(\theta)}{b_{2}+K_{V}^{*}(\theta)+\beta I^{*}(\theta)+\beta_{A} A^{*}(\theta)+\beta_{D} D^{*}(\theta)}, \\
& R^{*}(\theta)=\frac{\left(\tau_{0}+K_{\xi}^{*}(\theta)\right) I^{*}(\theta)+\tau_{0} A^{*}(\theta)+V_{0}^{*}(\theta)}{b_{2}+\eta} \\
&+\frac{K_{V}^{*}(\theta)}{b_{2}+\eta} S^{*}(\theta) \\
&=\frac{\left(\tau_{0}+K_{\xi}^{*}(\theta)\right) I^{*}(\theta)+\tau_{0} A^{*}(\theta)+V_{0}^{*}(\theta)}{b_{2}+\eta} \\
&+\frac{K_{V}^{*}(\theta)}{b_{2}+\eta} \quad b_{1}-V_{0}^{*}(\theta)+\eta R^{*}(\theta) \\
&+\frac{b_{2}+K_{V}^{*}(\theta)+I^{*}(\theta)+\beta_{A} A^{*}(\theta)+\beta_{D} D^{*}(\theta)}{b^{*}}
\end{aligned}
$$

lead to

$$
\begin{aligned}
& (1 \\
& \left.-\frac{K_{V}^{*}(\theta) \eta}{\left(b_{2}+\eta\right)\left(b_{2}+K_{V}^{*}(\theta)+\beta I^{*}(\theta)+\beta_{A} A^{*}(\theta)+\beta_{D} D^{*}(\theta)\right)}\right) \\
& \cdot R^{*}(\theta)=\frac{1}{b_{2}+\eta}\left[\left(\tau_{0}+K_{\xi}^{*}(\theta)\right) I^{*}(\theta)+\tau_{0} A^{*}(\theta)+V_{0}^{*}(\theta)\right. \\
& \left.+\frac{K_{V}^{*}(\theta)\left(b_{1}-V_{0}^{*}(\theta)\right)}{\left(b_{2}+K_{V}^{*}(\theta)+\beta I^{*}(\theta)+\beta_{A} A^{*}(\theta)+\beta_{D} D^{*}(\theta)\right)}\right]
\end{aligned}
$$

which may be simplified as $R^{*}(\theta)=N_{R}^{*}(\theta) / D_{R}^{*}(\theta) ; \forall \theta \in$ $\left[0, T_{D}^{*}\right)$. Thus, Property (iii) follows.

On the other hand, the linearized error of the trajectory solution with respect to an equilibrium trajectory is defined by

$$
\begin{aligned}
& \dot{\tilde{x}}(t)=\mathbf{A}^{*} \tilde{x}(t), \\
& \tilde{x}\left(t_{i}\right)=\left(\mathbf{I}_{6}-\mathbf{M}^{*}\right) \tilde{x}\left(t_{i}^{-}\right) ; \\
& \forall t \in\left[t_{i}, t_{i+1}\right), \forall t_{i} \in \operatorname{Imp} D,
\end{aligned}
$$

where $\tilde{x}\left(0^{-}\right)=\tilde{x}_{0}$ and $\mathbf{M}^{*}$ are $\mathbf{R}^{6} \times \mathbf{R}^{6}$ matrix taking account of the impulses, where $\left(\mathbf{M}^{*}\right)_{55}=\rho_{D}^{*}$ as $\rho_{D}(t) \rightarrow \rho_{D}^{*}$ as $t \rightarrow \infty$ and its remaining entries being zero. The following result, concerning the disease-free and endemic equilibrium points, holds if the control gains converge to constant values and $\rho_{D}^{*}=0$.

Theorem 5. Assume that $V_{0}(t) \rightarrow V_{0}, K_{V}(t) \rightarrow K_{V}^{*}, K_{\xi}(t) \rightarrow$ $K_{\xi}^{*}$ and $\rho_{D}\left(t_{i}\right) \rightarrow \rho_{D}^{*}=0$, and $\left(t_{i+1}-t_{i}\right) \rightarrow T_{D}^{*}$ as $t, t_{i}(\epsilon$ $\operatorname{Imp} D) \rightarrow \infty$. Then, the following properties hold:

(i) There is a unique disease-free equilibrium point satisfying

$$
\begin{aligned}
x_{d f}^{*} & :=\lim _{t \rightarrow \infty} x(t)=\left(S_{d f}^{*}, E_{d f}^{*}, I_{d f}^{*}, A_{d f}^{*}, D_{d f}^{*}, R_{d f}^{*}\right)^{T} \\
& =\left(S_{d f}^{*}, 0,0,0,0, R_{d f}^{*}\right)^{T}
\end{aligned}
$$

with

$$
\begin{aligned}
S_{d f}^{*} & =\frac{b_{2}\left(b_{1}-V_{0}\right)+\eta b_{1}}{b_{2}\left(b_{2}+\eta+K_{V}^{*}\right)}=\frac{b_{1}+\eta N_{d f}^{*}-V_{0}}{b_{2}+\eta+K_{V}^{*}}, \\
R_{d f}^{*} & =\frac{b_{2} V_{0}+K_{V}^{*} b_{1}}{b_{2}\left(b_{2}+\eta+K_{V}^{*}\right)}=\frac{K_{V}^{*} N_{d f}^{*}+V_{0}}{b_{2}+\eta+K_{V}^{*}} \\
& =\frac{K_{V}^{*} S_{d f}^{*}+V_{0}}{b_{2}+\eta}=N_{d f}^{*}-S_{d f}^{*}
\end{aligned}
$$

leading to an associated limit total population

$$
\bar{N}_{d f}^{*}=N_{d f}^{*}=S_{d f}^{*}+R_{d f}^{*}=\frac{b_{1}}{b_{2}}
$$


under a vaccination disease-free limiting control $V_{d f}^{*}=$ $V_{0}+K_{V}^{*} S_{d f}^{*}$ and a zero antiviral treatment control. (ii) There exists some large enough threshold $\beta_{\text {cend }}$ such that if $\beta>\beta_{\text {cend }}$ then there is a unique endemic equilibrium point with all its components being positive such that

$$
\begin{aligned}
& N_{d f}^{*}>S_{\text {end }}^{*}=\frac{\mu\left(b_{2}+\gamma\right)\left(b_{2}+\tau_{0}\right)\left(b_{2}+\alpha+\tau_{0}+K_{\xi}^{*}\right)}{\beta\left(\gamma p\left(b_{2}+\tau_{0}\right)\left(\mu+\beta_{D r}\left(b_{2}+\alpha\right)\right)+\gamma(1-p)\left(b_{2}+\alpha+\tau_{0}+K_{\xi}^{*}\right)\left(\beta_{A r} \mu+\beta_{D r} b_{2}\right)\right)}>0 \\
& S_{\text {end }}^{*}=\frac{b_{2}+\gamma}{\beta\left(C_{I}+\beta_{A r} C_{A}+\beta_{D r} C_{D}\right)}=\frac{b_{1}-V_{0}+\eta R_{\text {end }}^{*}}{b_{2}+K_{V}^{*}+\beta\left(C_{I}+\beta_{A r} C_{A}+\beta_{D r} C_{D}\right) E_{\text {end }}^{*}}, \\
& R_{\text {end }}^{*}=\frac{\left(\left(\tau_{0}+K_{\xi}^{*}\right) C_{I}+\tau_{0} C_{A}\right) E_{\text {end }}^{*}+V_{0}+K_{V}^{*} S_{\text {end }}^{*}}{b_{2}+\eta}, \\
& \bar{N}_{\text {end }}^{*}=\frac{\left(\tau_{0}+K_{\xi}^{*}\right) I_{\text {end }}^{*}+\tau_{0} A_{\text {end }}^{*}+V_{0}}{b_{2}+\eta}+\left(1+\frac{K_{V}^{*}}{b_{2}+\eta}\right) S_{\text {end }}^{*}+\left(C_{I}+C_{A}+C_{D}+1\right) E_{\text {end }}^{*}
\end{aligned}
$$

where $\beta_{A r}=\beta_{A} / \beta$ and $\beta_{D r}=\beta_{D} / \beta$ are relative disease coefficient transmission rates of the asymptomatic infectious and lying infective corpses with respect to the symptomatic infectious one, and

$$
\begin{aligned}
C_{I} & =\frac{\gamma p}{b_{2}+\alpha+\tau_{0}+K_{\xi}^{*}}, \\
C_{A} & =\frac{\gamma(1-p)}{b_{2}+\tau_{0}}
\end{aligned}
$$

$$
C_{D}=\frac{1}{\mu}\left[\frac{\left(b_{2}+\alpha\right) \gamma p}{b_{2}+\alpha+\tau_{0}+K_{\xi}^{*}}+\frac{b_{2} \gamma(1-p)}{b_{2}+\tau_{0}}\right] .
$$

(iii) The disease-free and endemic equilibrium dynamics matrices are, respectively, given by

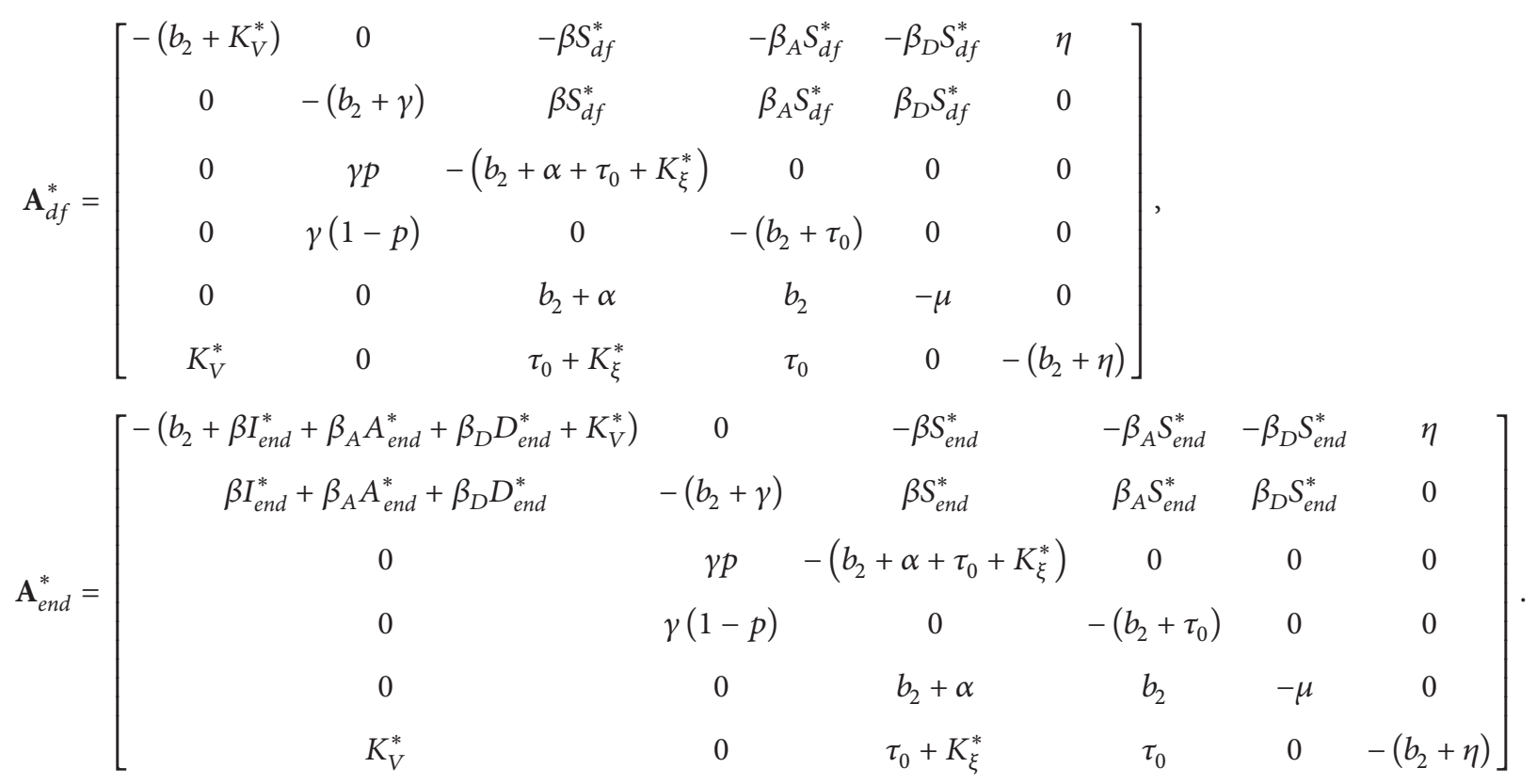


Note that the endemic equilibrium linearized dynamics can also be described equivalently by

$$
\bar{A}_{\text {end }}^{*}=\left[\begin{array}{cccccc}
-\left(b_{2}+K_{V}^{*}\right) & 0 & -(\beta+1) S_{\text {end }}^{*} & -\left(\beta_{A}+1\right) S_{\text {end }}^{*}-\left(\beta_{D}+1\right) S_{\text {end }}^{*} & \eta \\
0 & -\left(b_{2}+\gamma\right) & (\beta+1) S_{\text {end }}^{*} & \left(\beta_{A}+1\right) S_{\text {end }}^{*} & \left(\beta_{D}+1\right) S_{\text {end }}^{*} & 0 \\
0 & \gamma p & -\left(b_{2}+\alpha+\tau_{0}+K_{\xi}^{*}\right) & 0 & 0 & 0 \\
0 & \gamma(1-p) & 0 & -\left(b_{2}+\tau_{0}\right) & 0 & 0 \\
0 & 0 & b_{2}+\alpha & b_{2} & -\mu & 0 \\
K_{V}^{*} & 0 & \tau_{0}+K_{\xi}^{*} & \tau_{0} & 0 & -\left(b_{2}+\eta\right)
\end{array}\right] .
$$

(iv) If $\rho_{D}^{*} \in(0,1)$ then the endemic equilibrium steady state $x_{\text {end }}^{*}(\theta)$ for $\theta \in\left[0, T_{D}^{*}\right)$ is periodic of period $T_{D}^{*}$ leading to a matrix of dynamics $\mathbf{A}_{\text {end }}^{*}:\left[0, T_{D}^{*}\right) \rightarrow$ $\mathbf{R}^{6 \times 6}$ with $\mathbf{A}_{\text {end }}^{*}\left(T_{D}^{*}\right)=\mathbf{A}_{\text {end }}^{*}(0)$ and $\mathbf{A}_{\text {end }}^{*}\left(T_{D}^{*-}\right)=$ $\mathbf{A}_{\text {end }}^{*}\left(0^{-}\right) \neq \mathbf{A}_{\text {end }}^{*}(0)$. Equations (45)-(47) and (50)-(51) remain valid with the change $x_{\text {end }}^{*} \rightarrow x_{\text {end }}^{*}(\theta)$ and the corresponding changes in the two first rows of (50) and (51) for $\theta \in\left[0, T_{D}^{*}\right)$.

If the limit control gains $V_{0}^{*}(\cdot), K_{V}^{*}(\cdot)$, and $K_{\xi}^{*}(\cdot)$ are periodic functions of period $T_{D}^{*}$ then the disease-free equilibrium state has periodic susceptible and immune components defined as in Property (i) with the replacements $K_{V}^{*} \rightarrow K_{V}^{*}(\theta)$ and $K_{\xi}^{*} \rightarrow$ $K_{\xi}^{*}(\theta)$ for $\theta \in\left[0, T_{D}^{*}\right)$ and $\mathbf{A}_{d f}^{*}:\left[0, T_{D}^{*}\right) \rightarrow \mathbf{R}^{6 \times 6}$ in (49). In this case, the endemic equilibrium state, if it exists, is also periodic of period $T_{D}^{*}$.

Proof. The disease-free equilibrium point is obtained directly from (1) to (7) from the constraints $E_{\mathrm{df}}^{*}=I_{\mathrm{df}}^{*}=A_{\mathrm{df}}^{*}=D_{\mathrm{df}}^{*}=0$ and it is seen to be trivially unique. The Jacobian matrix of the linearized system at such a disease-free equilibrium point is (49). The proof of Property (i) follows directly. To prove the existence of an endemic equilibrium point (Property (ii)) some calculations are now performed to see the compatibility of the model with the existence of an equilibrium with exposed subpopulation $E_{\text {end }}^{*}>0$ implying the remaining subpopulations to be nonnegative. Direct calculations by zeroing in (3) to (5) the time derivatives of the subpopulations by taking into account (7)-(8) yield

$$
\begin{aligned}
& E_{\text {end }}^{*}>0 \Longleftrightarrow I_{\text {end }}^{*}=C_{I} E_{\text {end }}^{*}>0, \\
& E_{\text {end }}^{*}>0 \Longleftrightarrow A_{\text {end }}^{*}=C_{A} E_{\text {end }}^{*}>0, \\
& E_{\text {end }}^{*}>0 \Longleftrightarrow D_{\text {end }}^{*}=C_{D} E_{\text {end }}^{*}>0
\end{aligned}
$$

with the above constants defined in (48). From (2), one gets if $E_{\text {end }}^{*}>0$ implying that $I_{\text {end }}^{*}>0$ that (44) holds since

$$
\begin{aligned}
& E_{\text {end }}^{*}>0 \Longleftrightarrow \\
& {\left[\left(I_{\text {end }}^{*}>0\right) \wedge\left(A_{\text {end }}^{*}>0\right) \wedge\left(D_{\text {end }}^{*}>0\right)\right] \Longrightarrow} \\
& S_{\text {end }}^{*}=\frac{b_{2}+\gamma}{\beta\left(C_{I}+\beta_{A r} C_{A}+\beta_{D r} C_{D}\right) E_{\text {end }}^{*}} E_{\text {end }}^{*} .
\end{aligned}
$$

This proves the first part of Property (ii) since $N_{\text {end }}^{*}<N_{\mathrm{df}}^{*}$. Now, note from (44) that if $\beta \leq \beta_{\text {cend }}$ for a small enough threshold $\beta_{\text {cend }}$ for some existing small enough threshold $\beta_{\text {cend }}$, then $S_{\text {end }}^{*} \geq N_{\text {end }}^{*}$ from (44). This implies that $S_{\text {end }}^{*}>0$ from (44) but $E_{\text {end }}^{*} \leq 0$ (then either the endemic equilibrium point does not exist, since it has negative components, or it coincides with the disease-free one) since (46) leads to $E_{\text {end }}^{*}>$ 0 and $S_{\text {end }}^{*}>0$ implies $R_{\text {end }}^{*}>0$ and $R_{\text {end }}^{*}<0$ with $S_{\text {end }}^{*}>0$ if and only if $E_{\text {end }}^{*}<0$. Therefore, $E_{\text {end }}^{*}>0 \Leftrightarrow\left(N_{\text {end }}^{*}>S_{\text {end }}^{*}>0\right)$ if and only if $\beta>\beta_{\text {cend }}$. Now, summing up (1), (2), and (6), by taking into account (7)-(8) at the endemic equilibrium point yield (45)-(47) since

$$
\begin{gathered}
b_{2}\left(S_{\text {end }}^{*}+R_{\text {end }}^{*}\right) \\
=b_{1}+\left[\left(\tau_{0}+K_{\xi}^{*}\right) C_{I}+\tau_{0} C_{A}-b_{2}-\gamma\right] E_{\text {end }}^{*}, \\
R_{\text {end }}^{*}-\frac{K_{V}^{*} S_{\text {end }}^{*}+V_{0}}{b_{2}+\eta}=\frac{\left(\tau_{0}+K_{\xi}^{*}\right) I_{\text {end }}^{*}+\tau_{0} A_{\text {end }}^{*}}{b_{2}+\eta} \\
=\frac{\left(\tau_{0}+K_{\xi}^{*}\right) C_{I}+\tau_{0} C_{A}}{b_{2}+\eta} E_{\text {end }}^{*}, \\
\bar{N}_{\text {end }}^{*}=R_{\text {end }}^{*}+S_{\text {end }}^{*}+\left(I_{\text {end }}^{*}+A_{\text {end }}^{*}+D_{\text {end }}^{*}+E_{\text {end }}^{*}\right) \\
=\frac{\left(\tau_{0}+K_{\xi}^{*}\right) I_{\text {end }}^{*}+\tau_{0} A_{\text {end }}^{*}+V_{0}}{b_{2}+\eta} \\
\quad+\left(1+\frac{K_{V}^{*}}{b_{2}+\eta}\right) S_{\text {end }}^{*}+\left(C_{I}+C_{A}+C_{D}+1\right) E_{\text {end }}^{*}
\end{gathered}
$$

which completes the proof of Property (ii). The proof of Property (iii) is direct by taking the respective Jacobian matrices at the disease-free equilibrium point and the endemic equilibrium. The respective Jacobian matrices are (49) and (50). The use of (51), replacing (50), as the matrix of linearized dynamics around the endemic equilibrium point is legitimated via the identity:

$$
\begin{aligned}
& \left(\beta I_{\text {end }}^{*}+\beta_{A} A_{\text {end }}^{*}+\beta_{D} D_{\text {end }}^{*}\right) S_{\text {end }}^{*} \\
& =\left[\begin{array}{lll}
\beta S_{\text {end }}^{*} & \beta_{A} S_{\text {end }}^{*} & \beta_{D} S_{\text {end }}^{*}
\end{array}\right]\left[\begin{array}{c}
I_{\text {end }}^{*} \\
A_{\text {end }}^{*} \\
D_{\text {end }}^{*}
\end{array}\right] .
\end{aligned}
$$


Property (iv) follows directly from Property (iii) and Theorem 4 with the replacement $x_{\text {end }}^{*} \rightarrow x_{\text {end }}^{*}(\theta)$ and $C_{I}=C_{I}(\theta)$ and $C_{D}=C_{D}(\theta)$ in (48) for $\theta \in\left[0, T_{D}^{*}\right)$ and, eventually, $S_{\mathrm{df}}^{*} \rightarrow S_{\mathrm{df}}^{*}(\theta)$ and $R_{\mathrm{df}}^{*} \rightarrow R_{\mathrm{df}}^{*}(\theta)$ if the control gains converge to periodic values of period $T_{D}^{*}$.

Theorem 5 is useful for the study under linearization of the solution trajectories around the disease-free equilibrium point if $\rho_{D}^{*}=0$ under limit gains of the other controls. However, if the above limit gain is nonzero and less than one, then the trajectory solutions are asymptotically periodic. It is also proved the existence and uniqueness of the endemic equilibrium point if the coefficient transmission rates exceed a certain minimum threshold $\beta_{\text {cend }}$. It is also deduced from the disease-free equilibrium expressions that the susceptible disease-free equilibrium numbers can be decreased, and correspondingly the immune equilibrium numbers increased, by increasing the constant vaccination and/or the linear vaccination gains.

A constraint for the endemic equilibrium solution, if it exists, is discussed and given in the subsequent result. The existence constraints are easy to test under the form $S_{\text {end }}^{*}(\theta)<$ $b_{1} / b_{2}-V_{0}^{*}(\theta), \forall \theta \in\left[0, T_{D}^{*}\right)$, or some equivalent constraints, where $T_{D}^{*}$ is the limit interculling action period.

Theorem 6. Assume that $\left(t_{i+1}-t_{i}\right) \rightarrow T_{D}^{*}, \rho_{D}\left(n T_{D}^{*}\right) \rightarrow \rho_{D}^{*} \epsilon$ $[0,1), V_{0}\left(n T_{D}^{*}+\theta\right) \rightarrow V_{0}^{*}(\theta), K_{V}\left(n T_{D}^{*}+\theta\right) \rightarrow K_{V}^{*}(\theta)$ and $K_{\xi}\left(n T_{D}^{*}+\theta\right) \rightarrow K_{\xi}^{*}(\theta)$ as $n \rightarrow \infty, \forall \theta \in\left[0, T_{D}^{*}\right]$ as $t \rightarrow \infty$, $t_{i}(\in \operatorname{Imp} D) \rightarrow \infty$, and $n\left(\in \mathbf{Z}_{+}\right) \rightarrow \infty$. Then, the following properties hold:

(i) The endemic equilibrium state $x_{\text {end }}^{*}=x_{\text {end }}^{*}(\theta)$ for $\theta \in\left[0, T_{D}^{*}\right]$, being a point if $\rho_{D}^{*}=0$, equivalently if card $\operatorname{Imp} D<\aleph_{0}$ (i.e., it is finite), and a periodic limit oscillation if $\rho_{D}^{*} \in(0,1]$ has the subsequent components:

$$
\begin{aligned}
& S_{\text {end }}^{*}(\theta)=\frac{B_{A} B_{I}(\theta) B_{E}}{\beta f+\beta_{A} f_{A}(\theta)+\beta_{D} f_{D}(\theta)} \\
& =\frac{b_{2}+\gamma}{\beta\left(C_{I}(\theta)+\beta_{A r} C_{A}+\beta_{D r} C_{D}(\theta)\right)}, \\
& E_{\text {end }}^{*}(\theta)=B_{A} B_{I}(\theta) C_{0}(\theta) \\
& \cdot\left(B_{R}\left(S_{d f}^{*}(\theta)-S_{\text {end }}^{*}(\theta)\right)+K_{V}^{*}(\theta) S_{d f}^{*}\right), \\
& I_{\text {end }}^{*}(\theta)=C_{I}(\theta) E_{\text {end }}^{*}(\theta)=f C_{0}(\theta) \\
& \cdot\left(B_{R}\left(S_{d f}^{*}(\theta)-S_{\text {end }}^{*}(\theta)\right)+K_{V}^{*}(\theta) S_{d f}^{*}(\theta)\right), \\
& A_{\text {end }}^{*}(\theta)=C_{A} E_{\text {end }}^{*}(\theta)=f C_{0}(\theta) \\
& \cdot\left(B_{R}\left(S_{d f}^{*}(\theta)-S_{\text {end }}^{*}(\theta)\right)+K_{V}^{*}(\theta) S_{d f}^{*}(\theta)\right), \\
& D_{\text {end }}^{*}(\theta)=C_{D}(\theta) E_{\text {end }}^{*}(\theta)=f_{D}(\theta) C_{0}(\theta) \\
& \cdot\left(B_{R}\left(S_{d f}^{*}(\theta)-S_{\text {end }}^{*}(\theta)\right)+K_{V}^{*} S_{d f}^{*}(\theta)\right),
\end{aligned}
$$

$$
\begin{aligned}
& R_{\text {end }}^{*}(\theta)=\left(S_{d f}^{*}(\theta)-S_{\text {end }}^{*}(\theta)\right) \\
& +\left(R_{d f}^{*}(\theta)-\left(E_{\text {end }}^{*}(\theta)+\frac{\mu}{b_{2}} D_{\text {end }}^{*}(\theta)\right)\right) ; \\
& \forall \theta \in\left[0, T_{D}^{*}\right] .
\end{aligned}
$$

Being real constants if $\rho_{D}^{*}=0$, where

$$
\begin{aligned}
B_{A} & =b_{2}+\tau_{0} ; \\
B_{I}(\theta) & =b_{2}+\tau_{0}+\alpha+K_{\xi}^{*}(\theta), \\
B_{R} & =b_{2}+\eta ; \\
B_{E} & =b_{2}+\gamma \\
f & =\gamma p B_{A} ; \\
f_{A}(\theta) & =\gamma(1-p) B_{I}(\theta), \\
f_{D}(\theta) & =\frac{1}{\mu}\left(b_{2} f_{A}(\theta)+\left(b_{2}+\alpha\right) f\right) \\
& =\frac{\gamma}{\mu}\left(b_{2}(1-p) B_{I}(\theta)+p\left(b_{2}+\alpha\right) B_{A}\right), \\
C_{0}(\theta) & =\frac{b_{2}}{b_{2} B_{A} B_{I}(\theta)\left(\gamma+B_{R}\right)+\eta \mu f_{D}(\theta)} ;
\end{aligned}
$$

where $C_{I}=C_{I}(\theta)$ and $C_{D}=C_{D}(\theta)$ in (48) since $K_{\xi}^{*}=K_{\xi}^{*}(\theta)$ for $\theta \in\left[0, T_{D}^{*}\right)$. If $S_{\text {end }}^{*}(\theta)<\left(\left(B_{R}+\right.\right.$ $\left.\left.K_{V}^{*}(\theta)\right) / B_{R}\right) S_{d f}^{*}(\theta)=\left(1+K_{V}^{*}(\theta) /\left(b_{2}+\eta\right)\right) S_{d f}^{*}(\theta) ; \forall \theta \epsilon$ $\left[0, T_{D}^{*}\right)$, then the endemic equilibrium state exists, while being distinct of the disease-free equilibrium state. This existence condition of the endemic equilibrium state is equivalent to $S_{\text {end }}^{*}(\theta)<b_{1} / b_{2}-V_{0}^{*}(\theta) ; \forall \theta \in\left[0, T_{D}^{*}\right)$.

If $S_{\text {end }}^{*}(\theta)>\left(1+K_{V}^{*}(\theta) /\left(b_{2}+\eta\right)\right) S_{d f}^{*}(\theta) ; \forall \theta \in\left[0, T_{D}^{*}\right)$ then the endemic equilibrium state does not exist in the sense that it has some negative components. On the contrary, the opposed condition

$$
\begin{aligned}
S_{\text {end }}^{*}(\theta) & <\left(1+\frac{K_{V}^{*}(\theta)}{b_{2}+\eta}\right) S_{d f}^{*}(\theta) \\
& =\frac{b_{2}\left(b_{1}-V_{0}^{*}(\theta)\right)+\eta b_{1}}{b_{2}\left(b_{2}+\eta\right)} ; \quad \theta \in\left[0, T_{D}^{*}\right)
\end{aligned}
$$

yields the existence of such an endemic equilibrium state. In the case when the limit control gains are constant, the disease-free equilibrium state is an equilibrium point. If, in addition, $\rho_{D}^{*}=0$ then the endemic equilibrium solution, if it exists, is also an equilibrium point.

(ii)

$$
N_{\text {end }}^{*}(\theta)<N_{d f}^{*}=\frac{b_{1}}{b_{2}}
$$


and the dependence on $\theta \in\left[0, T_{D}^{*}\right)$ is removed in the case that the endemic equilibrium state is an equilibrium point.

Proof. The values of the components of the endemic equilibrium state follow by direct elementary calculations form (45) and (48) and have been verified under symbolic calculation with the Mathematica package. Note that in the general case when the control gains converge to periodic functions of period $T_{D}^{*}$ both the disease-free and endemic equilibrium solutions are periodic with such a period [see Theorem 5(iv)]. The endemic equilibrium exists while it is distinct from the disease-free one if (58) holds. To prove Property (ii), note by zeroing (1) to (4) and (6) while summing them up and the use of (7) at the disease-free and endemic equilibrium states that

$$
\begin{aligned}
N_{\text {end }}^{*}(\theta)=S_{\text {end }}^{*}(\theta)+R_{\text {end }}^{*}(\theta)+E_{\text {end }}^{*}(\theta)+I_{\text {end }}^{*}(\theta) & \\
+A_{\text {end }}^{*}(\theta)<N_{\mathrm{df}}^{*}=S_{\mathrm{df}}^{*}+R_{\mathrm{df}}^{*} & \text { } \\
& \forall \theta \in\left[0, T_{D}^{*}\right)
\end{aligned}
$$

since $\dot{N}(t)=-b_{2} N(t)+b_{1}-\alpha I(t) ; \forall t \in \mathbf{R}_{0+}$. Thus, since $\left(E_{\text {end }}^{*}(\theta)+I_{\text {end }}^{*}(\theta)+A_{\text {end }}^{*}(\theta)\right)>0 ; \forall \theta \in\left[0, T_{D}^{*}\right)$ implied by $E_{\text {end }}^{*}(\theta)>0, \forall \theta \in\left[0, T_{D}^{*}\right)$ if the endemic equilibrium state exists then $N_{\text {end }}^{*}(\theta)<N_{\mathrm{df}}^{*} ; \forall \theta \in\left[0, T_{D}^{*}\right)$. Property (ii) is proved.

Note from the components of the endemic equilibrium expressions given in Theorem 6(i) that the equilibrium number of the endemic susceptible increases while correspondingly those of all the infective subpopulations decrease as the limit antiviral control gain $K_{\xi}^{*}$ increases. This is an interesting tool to control the infection in the case that the endemic equilibrium exists and the disease-free one is unstable so unreachable in practice if the coefficient transmission rate is large enough exceeding the threshold $\beta_{\text {cend }}$ of Theorem 5.

Remark 7. Note that we can write the linearized equation around the endemic equilibrium state as

$$
\begin{aligned}
& \dot{\tilde{x}}(\theta)=\left\lceil\mathbf{A}_{\mathrm{df}}^{*}(\theta)+\left(\mathbf{A}_{\text {end }}^{*}(\theta)-\mathbf{A}_{\mathrm{df}}^{*}(\theta)\right)\right\rceil \tilde{x}(\theta) \\
&=\left\lceil\mathbf{A}_{\mathrm{df}}^{*}(\theta)+\left(\overline{\mathbf{A}}_{\mathrm{end}}^{*}(\theta)-\mathbf{A}_{\mathrm{df}}^{*}(\theta)\right)\right\rceil \tilde{x}(\theta) ; \\
& \forall \theta \in\left[0, T_{D}^{*}\right)
\end{aligned}
$$

with

$$
\tilde{x}(0)=\tilde{x}\left(T_{D}^{*}\right)=\left(1-\rho_{D}^{*}\right) \tilde{x}\left(T_{D}^{*-}\right)
$$

where

$$
\begin{aligned}
\overline{\mathbf{A}}_{\text {end }}^{*}(\theta)-\mathbf{A}_{\mathrm{df}}^{*}(\theta) \\
\quad=\left[\begin{array}{cccccc}
0 & 0 & -a_{13}(\theta) & -a_{14}(\theta) & -a_{15}(\theta) & 0 \\
0 & 0 & a_{13}(\theta) & a_{14}(\theta) & a_{15}(\theta) & 0 \\
& & & \mathbf{0}_{4 \times 6} & &
\end{array}\right]
\end{aligned}
$$

with

$$
\begin{aligned}
a_{13}(\theta) & =(\beta+1) S_{\text {end }}^{*}(\theta)-\beta S_{\mathrm{df}}^{*}(\theta), \\
a_{14}(\theta)=\left(\beta \beta_{A r}+1\right) S_{\text {end }}^{*}(\theta)-\beta \beta_{A r} S_{\mathrm{df}}^{*}(\theta), & \\
a_{15}(\theta)=\left(\beta \beta_{D r}+1\right) S_{\text {end }}^{*}(\theta)-\beta \beta_{D r} S_{\mathrm{df}}^{*}(\theta) ; & \forall \theta \in\left[0, T_{D}^{*}\right)
\end{aligned}
$$

since

$$
\begin{array}{r}
\mathbf{A}_{\text {end }}^{*}(\theta) x_{\text {end }}^{*}(\theta)=\overline{\mathbf{A}}_{\text {end }}^{*}(\theta) x_{\text {end }}^{*}(\theta) \\
=\left[\mathbf{A}_{\mathrm{df}}^{*}(\theta)+\left(\overline{\mathbf{A}}_{\text {end }}^{*}(\theta)-\mathbf{A}_{\mathrm{df}}^{*}(\theta)\right)\right] x_{\text {end }}^{*}(\theta) ; \\
\forall \theta \in\left[0, T_{D}^{*}\right)
\end{array}
$$

by using (49)-(51) and (55). If $\mathbf{A}_{\mathrm{df}}^{*}(\theta)$ is nonsingular then $\overline{\mathbf{A}}_{\text {end }}^{*}(\theta)=\mathbf{A}_{\mathrm{df}}^{*}(\theta)\left[\mathbf{I}_{6}+\mathbf{A}_{\mathrm{df}}^{*-1}(\theta)\left(\overline{\mathbf{A}}_{\text {end }}^{*}(\theta)-\mathbf{A}_{\mathrm{df}}^{*}(\theta)\right)\right]$ is also nonsingular if

$$
\begin{aligned}
\left\|\overline{\mathbf{A}}_{\text {end }}^{*}(\theta)-\mathbf{A}_{\mathrm{df}}^{*}(\theta)\right\|_{2}^{2} & =2\left[a_{13}^{2}(\theta)+a_{13}^{2}(\theta)+a_{15}^{2}(\theta)\right] \\
& <1 ; \quad \forall \theta \in\left[0, T_{D}^{*}\right) .
\end{aligned}
$$

Therefore, if $\mathbf{A}_{\mathrm{df}}^{*}(\theta)$ is a stability matrix (then, nonsingular) and (65) holds then $\overline{\mathbf{A}}_{\text {end }}^{*}(\theta)$ and $\mathbf{A}_{\text {end }}^{*}(\theta)$ are stability matrices.

The following results give easily testable sufficiency-type local instability and local stability tests for the endemic equilibrium point based on the stability properties of the disease-free matrix of dynamics of the linearized system about the disease-free equilibrium. The extension to the case of oscillatory periodic endemic equilibrium solution would follow "mutatis-mutandis."

Theorem 8. Assume that the control limits $V_{0}^{*}, K_{V}^{*}, K_{\xi}^{*}$, and $\rho_{D}^{*}=0$ exist and define the amounts

$$
\begin{aligned}
\vartheta & =\frac{1}{2}\left\|A_{d f}^{*^{-1}}\right\|_{1}\left\|\bar{A}_{\text {end }}^{*}-A_{d f}^{*}\right\|_{1}, \\
\kappa_{\vartheta} & =\left\|A_{d f}^{*^{-1}}\right\|_{1} \frac{b_{2}\left(b_{1}-V_{0}\right)+\eta b_{1}}{b_{2}\left(b_{2}+\eta+K_{V}^{*}\right)}\left[\frac{K_{V}^{*}}{b_{2}+\eta}(1\right. \\
& \left.\left.+\beta \max \left(1, \beta_{A r}, \beta_{D r}\right)\right)+1\right] .
\end{aligned}
$$

The following properties hold:

(i) The endemic equilibrium point exists and it is unstable if $A_{d f}^{*}$ is instability nonsingular matrix (i.e., it has at least one eigenvalue in $\operatorname{Re} s>0)$ and $\kappa_{9}<1 / 2$.

(ii) The endemic equilibrium point, provided that it exists, is locally asymptotically stable if $A_{d f}^{*}$ is a stability matrix and $\kappa_{9}<1 / 2$.

Proof. Elementary calculation yields $\bar{A}_{\text {end }}^{*}=A_{\mathrm{df}}^{*}\left[\mathbf{I}_{6}+\right.$ $\left.A_{\mathrm{df}}^{*-1}\left(\bar{A}_{\mathrm{end}}^{*}-A_{\mathrm{df}}^{*}\right)\right]$ if $A_{\mathrm{df}}^{*}$ is nonsingular. If, furthermore, $A_{\mathrm{df}}^{*}$ is instability matrix then $\bar{A}_{\text {end }}^{*}$ is also instability matrix if 
$1>\left\|A_{\mathrm{df}}^{*-1}\left(\bar{A}_{\mathrm{end}}^{*}-A_{\mathrm{df}}^{*}\right)\right\|_{1}$, which is equivalent to $\kappa_{\vartheta}<1 / 2$, from Banach's Perturbation Lemma [33], since $\bar{A}_{\text {end }}^{*}$ is nonsingular and the eigenvalues are continuous functions with respect to any matrix entry thus $\bar{A}_{\text {end }}^{*}$ is instability matrix. In the same way, if $A_{\mathrm{df}}^{*}$ is a stability matrix (then nonsingular) and $\kappa_{\vartheta}<1 / 2$ then $\bar{A}_{\text {end }}^{*}$ is nonsingular and then stable by similar reasoning.

It has to be pointed out that Theorem 10, which is stated and proved later on, establishes that both equilibrium points cannot be simultaneously stable. As a result, one concludes via Theorem 8 (ii) that if $A_{\mathrm{df}}^{*}$ is a stability matrix and $\kappa_{\vartheta}<1 / 2$ then the endemic equilibrium point does not exist. By linking this observation with Theorem 6(i), one concludes as well that $S_{\text {end }}^{*}>\left(1+K_{V}^{*} /\left(b_{2}+\eta\right)\right) S_{\mathrm{df}}^{*}$ and the only existing equilibrium point is the disease-free one which is globally asymptotically stable.

Theorem 8 can be reformulated for the use of $\ell_{\infty}$-norms by using the identity:

$$
\begin{aligned}
\left\|\bar{A}_{\text {end }}^{*}-A_{\mathrm{df}}^{*}\right\|_{\infty}= & \left|S_{\text {end }}^{*}+\beta\left(S_{\text {end }}^{*}-S_{\mathrm{df}}^{*}\right)\right| \\
& +\left|S_{\text {end }}^{*}+\beta \beta_{\text {Ar }}\left(S_{\text {end }}^{*}-S_{\mathrm{df}}^{*}\right)\right| \\
& +\left|S_{\text {end }}^{*}+\beta \beta_{\text {Dr }}\left(S_{\text {end }}^{*}-S_{\mathrm{df}}^{*}\right)\right|
\end{aligned}
$$

and for the use of $\ell_{2}$-norms by using the square root of the sum of the squares of the three right-hand-side terms in the above identity as replacement of it. A simple sufficient condition for the local stability of the disease-free equilibrium follows.

Theorem 9. Assume that $\beta$ is small enough according to $\beta<$ $\beta_{c d f}$ with respect to the threshold:

$$
\begin{aligned}
\beta_{c d f} & =\frac{1+\beta_{A r}+\beta_{D r}}{S_{d f}^{*}}\left[b_{2}+\min \left(\gamma, K_{V}^{*}-\eta\right)\right] \\
& =\frac{b_{2}\left(b_{2}+\eta+K_{V}^{*}\right)}{\left(1+\beta_{A r}+\beta_{D r}\right)\left[b_{2}\left(b_{1}-V_{0}\right)+\eta b_{1}\right]}\left(b_{2}\right. \\
& \left.+\min \left(\gamma, K_{V}^{*}-\eta\right)\right) .
\end{aligned}
$$

Thus, the disease-free equilibrium point is locally asymptotically stable provided that

$$
\begin{aligned}
& \alpha<\mu ; \\
& K_{V}^{*}>\eta-b_{2}, \\
& b_{2} \in\left(\operatorname { m a x } \left(\eta-K_{V}^{*}, K_{V}^{*}+2 \tau_{0}+K_{\xi}^{*}-\mu, \gamma(1-p)\right.\right. \\
&\left.\left.\quad-\tau_{0}, \gamma p-\alpha-\tau_{0}-K_{\xi}^{*}, 0\right), \frac{\mu-\alpha}{2}\right) .
\end{aligned}
$$

Proof. Note from (49) that $\mathbf{A}_{\mathrm{df}}^{*}$ is a stability matrix since $\operatorname{diag}\left(\mathbf{A}_{\mathrm{df}}^{*}\right)$ is a stability matrix and $\mathbf{A}_{\mathrm{df}}^{*}$ is diagonally row dominant if (68)-(69) hold.

Note that Theorem 9 can be combined with Theorem 5 in practical situations in the following sense. If the threshold $\beta<\beta_{\text {cdf }} \leq \beta_{\text {cend }}$ then the disease-free equilibrium is locally asymptotically stable and no endemic equilibrium point exists. If $\beta \geq \beta_{\text {cdf }} \geq \beta_{\text {cend }}$ then the endemic equilibrium point is locally asymptotically stable while the disease-free one is unstable. This local result has a global stability version as discussed in the following. The subsequent global stability result is proved in Appendix and it is based on the qualitative theory of differential equations in the sense that Lyapunov equation candidates are not used. The solution explicit formulas are not invoked to construct the proof but only the trajectory separating properties of eventually existing stable, semistable, or unstable limit cycles around equilibrium points are addressed and used.

Theorem 10 (global uniform asymptotic stability). Assume that $\rho_{D}(t) \rightarrow \rho_{D}^{*}=0$ as $t(\in \operatorname{Imp} D) \rightarrow \infty$. Thus, the following properties hold:

(i) If the disease-free equilibrium point is locally asymptotically stable while the endemic equilibrium state does not exist then the epidemic model is globally uniformly asymptotically stable and all the solution trajectories converge asymptotically to the disease-free equilibrium point.

(ii) If the disease-free equilibrium point is unstable and the endemic equilibrium state exists then the system is globally uniformly asymptotically stable and all the solution trajectories converge to the endemic equilibrium point.

(iii) The disease-free and the endemic equilibrium states cannot be simultaneously either stable or unstable.

\section{Numerical Simulations}

It is now presented a set of numerical simulation work. The parameters of the model are obtained from real data from a study of Ebola disease [29]. The recruitment rate and the natural average death rate are $b_{1}=b_{2}=1 /(70 \times 365) \times$ days $^{-1}$ while the disease transmission coefficients are $\beta=0.16, \beta_{A}=$ 0.05 , and $\beta_{D}=0.5\left(\times\right.$ days $\left.^{-1}\right)$, respectively. The average duration of the immunity period reflecting a transition from the recovered subpopulation to the susceptible subpopulation is determined by $1 / \eta=1000$ days, the average transition rate from the exposed to both infectious subpopulations is $\gamma=$ $1 / 15.8 \times$ days $^{-1}$, the average extra mortality of the symptomatic infectious is $\alpha=1 / 13.3 \times$ days $^{-1}$, the natural immune response is $\tau_{0}=1 / 12 \times$ days $^{-1}$, the fraction of the exposed subpopulation becoming symptomatic infectious one is $p=$ 0.9 , and the average duration of infection is $1 / \mu=20$ days. The initial conditions are given by $S(0)=1000 / 1050, E(0)=$ $10 / 1050, I(0)=30 / 1050, A(0)=D(0)=0$, and $R(0)=$ $10 / 1050$ so that the initial total living population is normalized to unity, $N(0)=S(0)+E(0)+I(0)+A(0)+D(0)+$ $R(0)=1$. Figure 1 displays the natural evolution of the disease in the absence of any external action. It is observed that the number of infective and infectious subpopulations increases implying an increase of infective corpses as well. The result of the natural evolution of the epidemics is the dead of individuals so that the total living population decreases with 


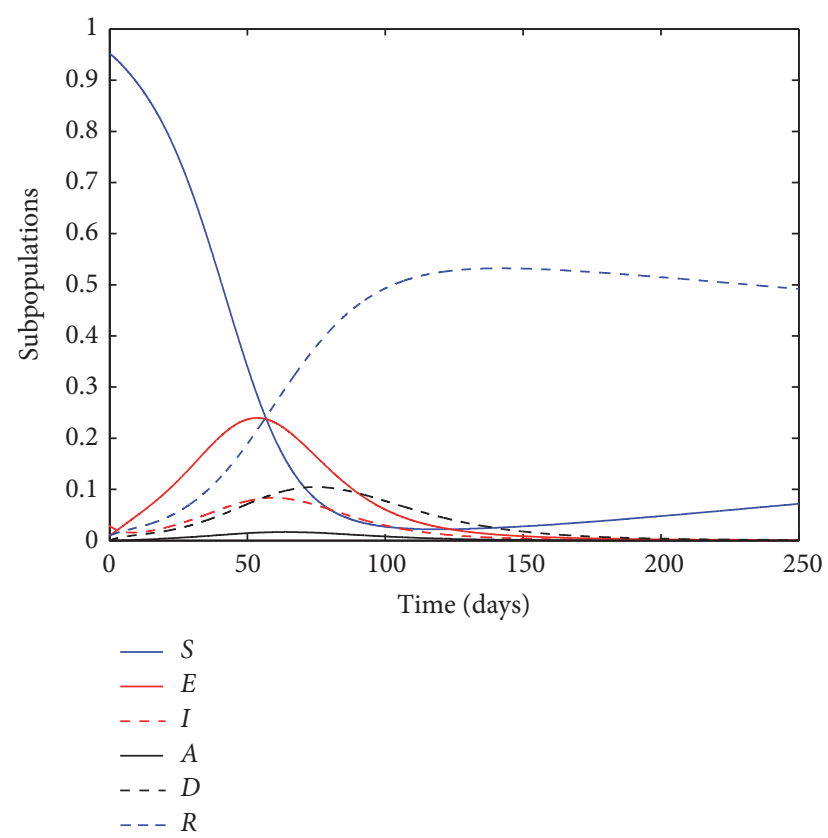

FIGURE 1: Natural evolution of the subpopulations.

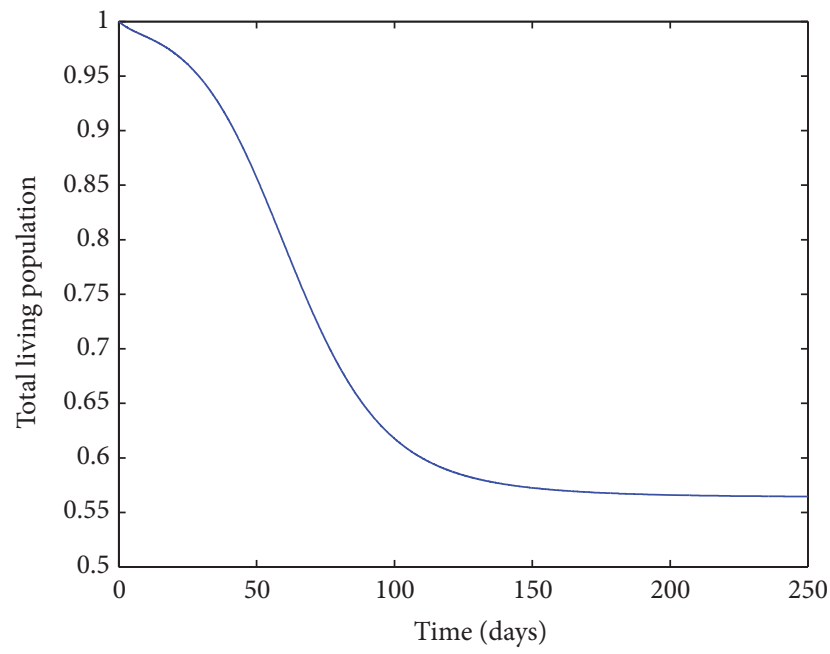

FIGURE 2: Natural evolution of the total alive population.

time as Figure 2 shows. After 250 days, the total living population is only $56.47 \%$ of the initial one. Three control mechanisms of fighting against Ebola have been considered in the previous subsections. The effect of these control policies is now illustrated through simulation examples. Initially, corpse culling (impulsive action on $D$ ) is considered as the only action to modify the natural behavior of the disease. Figures 3 and 4 show the effect of corpse culling on the system with different culling rates. In this way, Figure 3 considers the case when corpses are removed once daily at a rate of $\rho_{D}=0.1$ (i.e., $10 \%$ of corpses are removed daily) while Figure 4 shows the behavior of the system when the daily culling rate is $\rho_{D}=$ 0.8 .

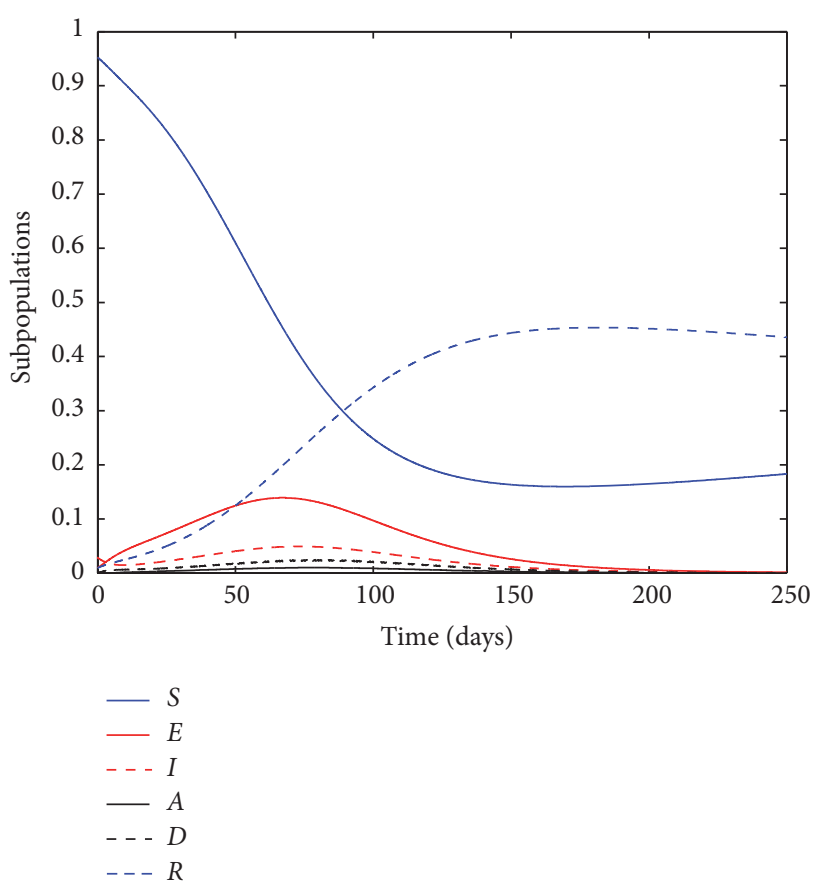

FIGURE 3: Evolution of the subpopulations with a daily culling rate of $\rho_{D}=0.1$.

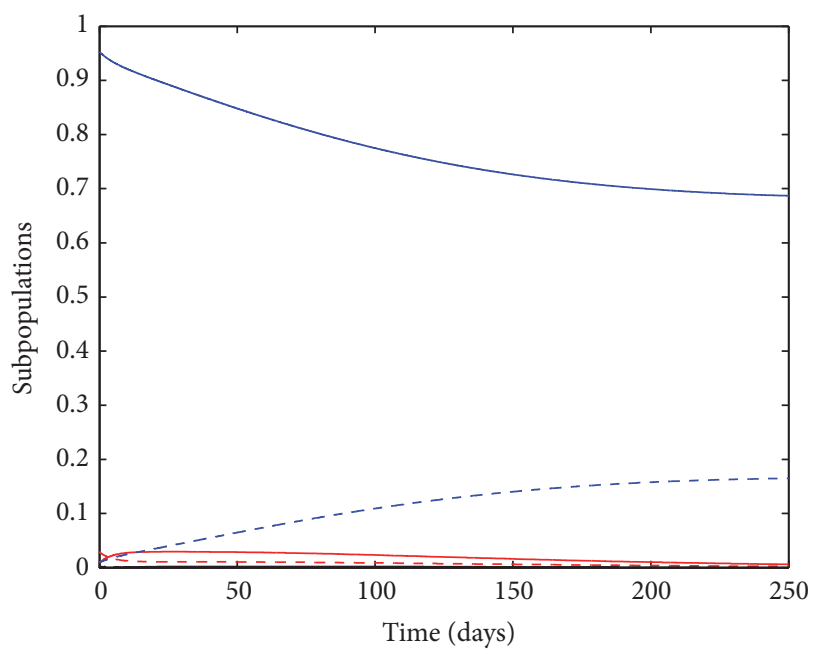

$$
\begin{aligned}
& -S \\
& -E \\
& --I \\
& -A \\
& --D \\
& ---R
\end{aligned}
$$

FIGURE 4: Evolution of the subpopulations with a daily culling rate of $\rho_{D}=0.8$.

It can be deduced from Figures 3 and 4 that corpse culling has a high impact on the evolution of the disease since all the infected populations reduce their peak values due to the application of culling. The direct consequence of this fact is that the number of casualties is reduced as Figures 5 and 6 reveal for the total living population. Therefore, when the culling rate is $\rho_{D}=0.1$, the total living population after 250 


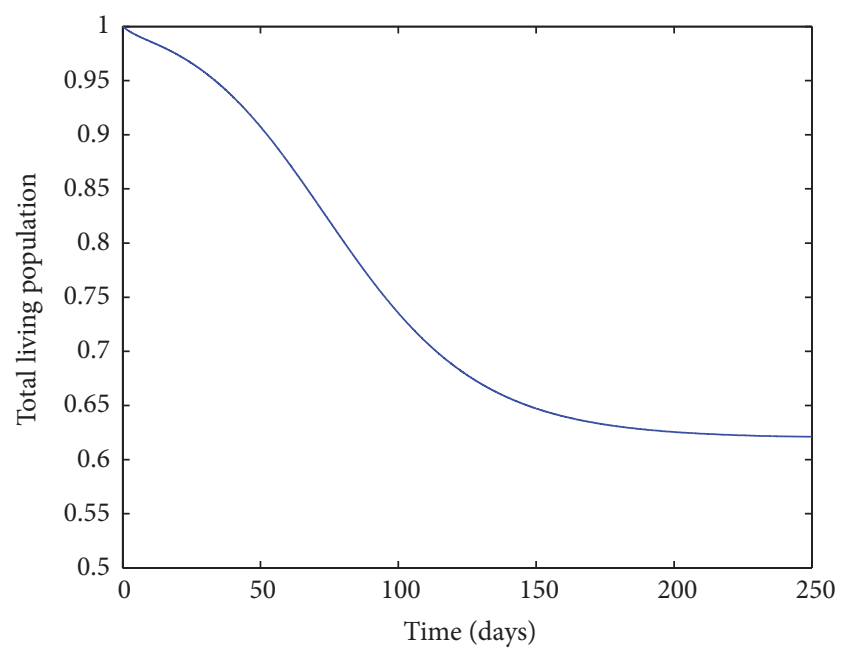

FIGURE 5: Evolution of the total alive population with a daily culling rate of $\rho_{D}=0.1$.

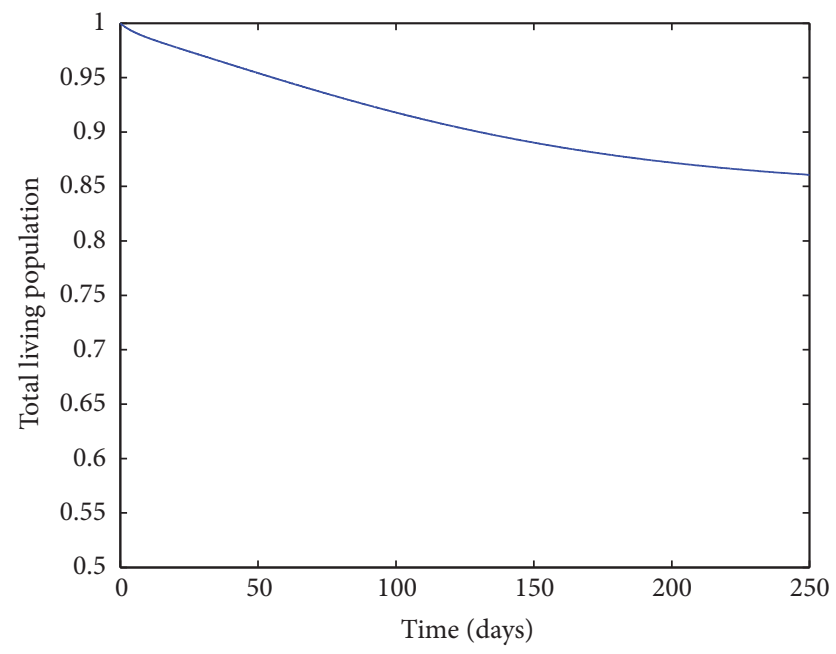

FIGURE 6: Evolution of the total alive population with a daily culling rate of $\rho_{D}=0.8$.

days is $62.10 \%$ of the initial one while when $\rho_{D}=0.8$ the total living population after 250 days is $86.07 \%$ of the initial one. On the other hand, Figures 7 and 8 show the effect of culling when applied every other day instead of daily.

If we now compare Figures 6 and 8 it can be noticed that the spacing of the culling action reduces the total living population after 250 days of epidemics. Thus, from Figures 5, 6, and 8 it is obtained the intuitive conclusion that it is recommendable to perform culling as frequently as possible with the highest possible rate. Hence, the proposed mathematical model (1)-(6) captures and illustrates the effect of culling in reality. Figures 9, 10, and 11 display the culling effort corresponding to the cases considered in Figures 3, 4, and 7 , respectively. The culling effort is higher during the first time instants for a higher culling rate while decreases afterwards. Thus, a greater number of corpses are removed initially, fact that reduces the number of deaths caused by the infection, which in turn reduces the number of new corpses.

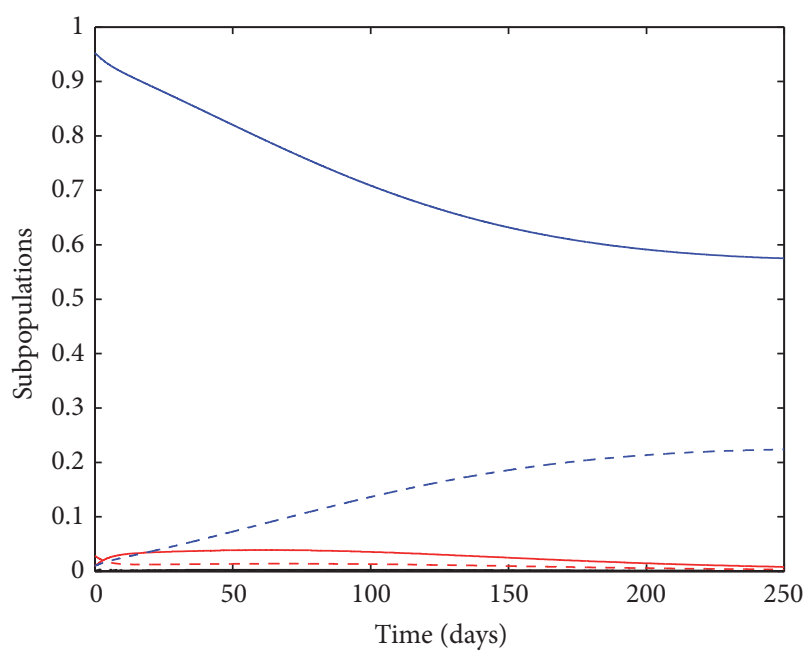

$$
\begin{aligned}
& -S \\
& -E \\
& --I \\
& -A \\
& --D \\
& ---R
\end{aligned}
$$

FIGURE 7: Evolution of the subpopulations with an every other day culling rate of $\rho_{D}=0.8$.

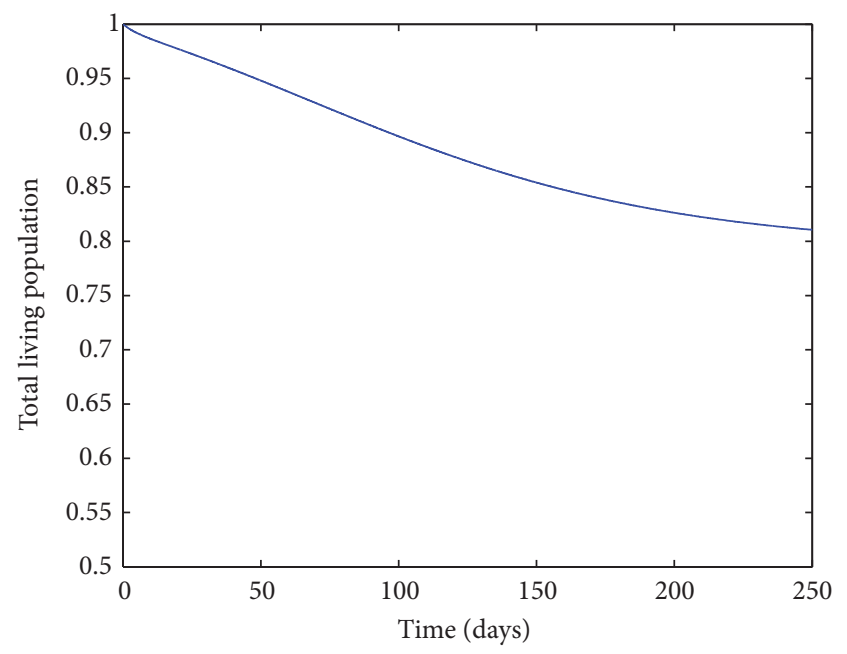

FIGURE 8: Evolution of the total alive population with an every other day culling rate of $\rho_{D}=0.8$.

As a consequence, the number of corpses to be removed reduces as time goes by. On the other hand, a smaller culling rate causes a peak in the culling effort during the evolution of the disease, as Figure 9 shows.

Furthermore, vaccination can also be used in addition to culling to fight against disease. In this way, Figures 1215 show the effect of a constant vaccination on the system when a culling rate of $\rho_{D}=0.1$ is also applied. The constant vaccination is expressed in both cases as a multiple of $b_{1}$, being of $V=V_{0}=0.2 b_{1}$ for Figures 12 and 13 and $V=V_{0}=0.8 b_{1}$ for Figures 14 and 15 .

It can be noted from Figures 5, 13, and 15 that the proposed constant vaccinations do not alter significantly the 


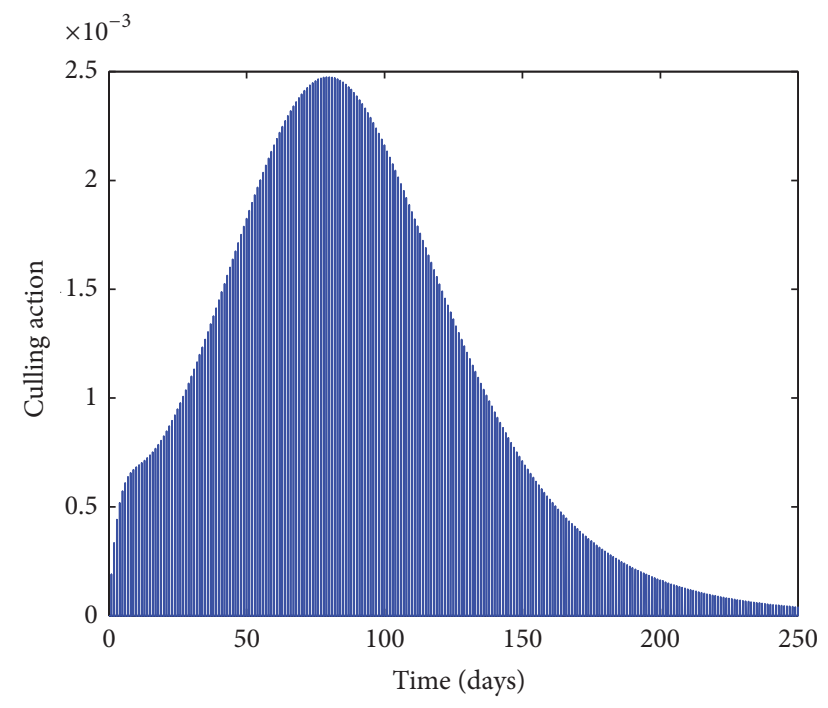

FIGURE 9: Culling effort $\rho_{D} D(t)$ with a daily culling rate of $\rho_{D}=0.1$.

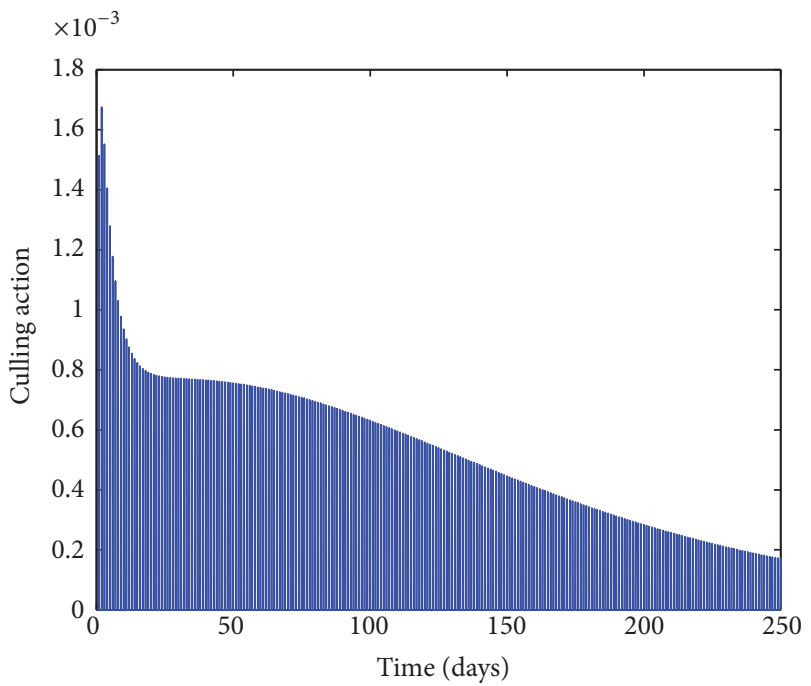

FIGURE 10: Culling effort $\rho_{D} D(t)$ with a daily culling rate of $\rho_{D}=0.8$.

behavior of the system where the culling action has been applied. This result points out that it may be difficult to tune the constant vaccination term $V_{0}$ in order to obtain an appropriate behavior of the controlled system. The proposed feedback vaccination given by (7) in Section 2 contributes to solving this tuning problem since it relates the vaccination effort to the actual evolution of the system in such a way that the amplitude of vaccination is calculated based on the current value of susceptible. Thus, Figures 16 and 17 show the system evolution when a feedback vaccination with a constant of $K_{V}=0.002$ is applied along with the constant vaccination term.

From Figures 12 and 16 we conclude that the feedback vaccination law calculated from the value of susceptible modifies significantly the behavior of the system while Figures 13 and 17 reveal that the total living population is largely improved by the action of feedback control. As a consequence, the main recommendation related to vaccination campaign design is to

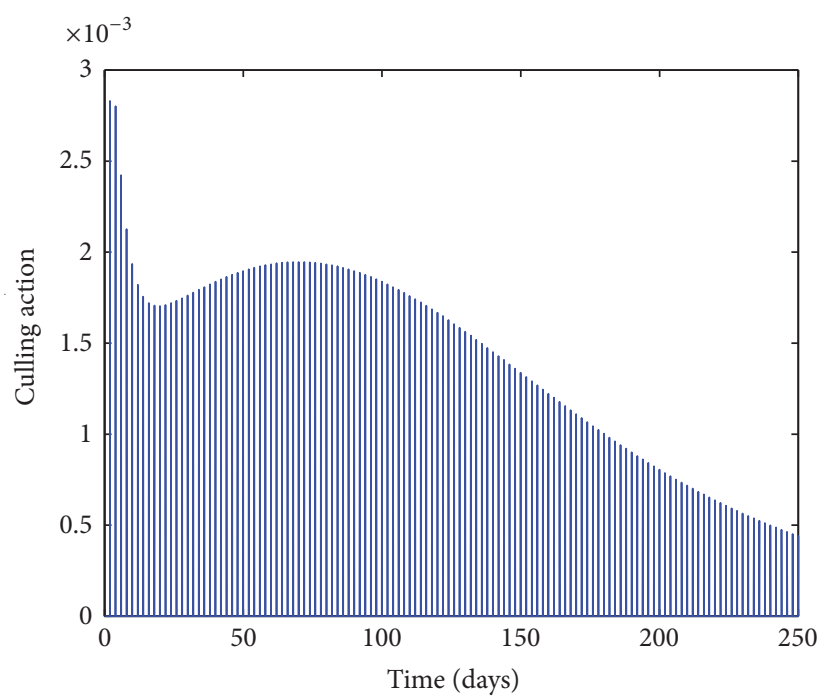

FIgURE 11: Culling effort $\rho_{D} D(t)$ with an every other culling rate of $\rho_{D}=0.8$.

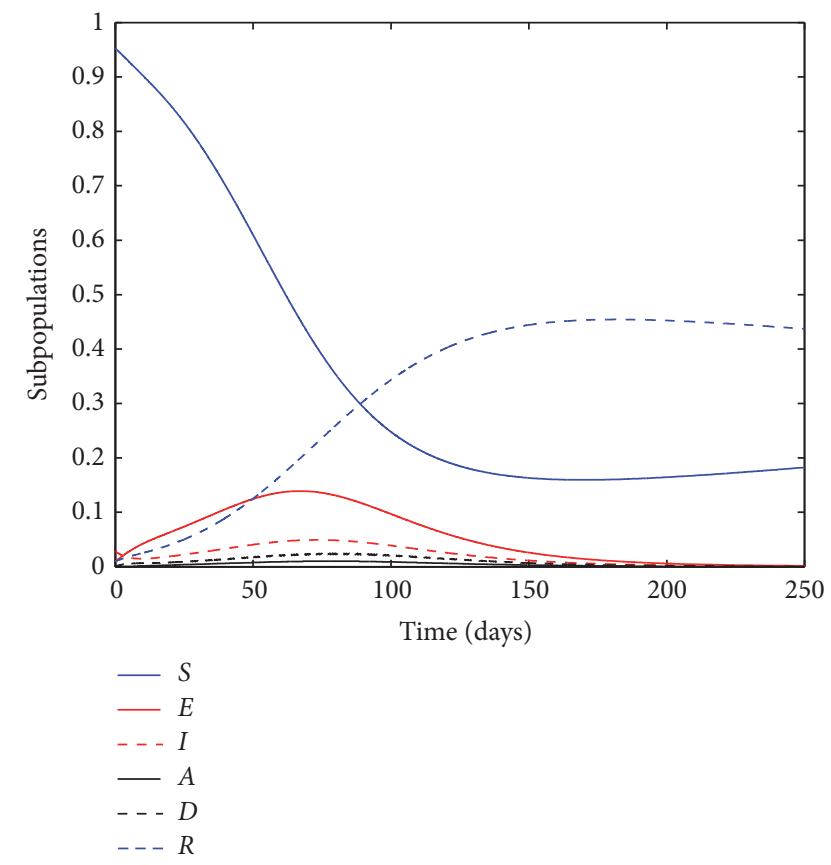

FIGURE 12: Evolution of the subpopulations with daily culling rate of $\rho_{D}=0.1$ and constant vaccination of $V=V_{0}=0.2 b_{1}$.

dynamically calculate the amount of vaccines to be applied by using the proposed feedback law (7). The vaccination control action is shown in Figure 18 while the culling effort corresponding to this case is depicted in Figure 19. It can be observed in Figure 19 that the culling action vanishes as a direct consequence of $D(t)$ tending to zero asymptotically. Therefore, the combination of culling and feedback vaccination allows stopping the mortality associated with the disease. Finally, we can also add antivirals to fight against Ebola. Antiviral action is given by (8) which is a feedback control law based on the symptomatic infectious subpopulation. In this case, we consider the constant linear value of $\xi(t)=K_{\xi} I(t)=$ 


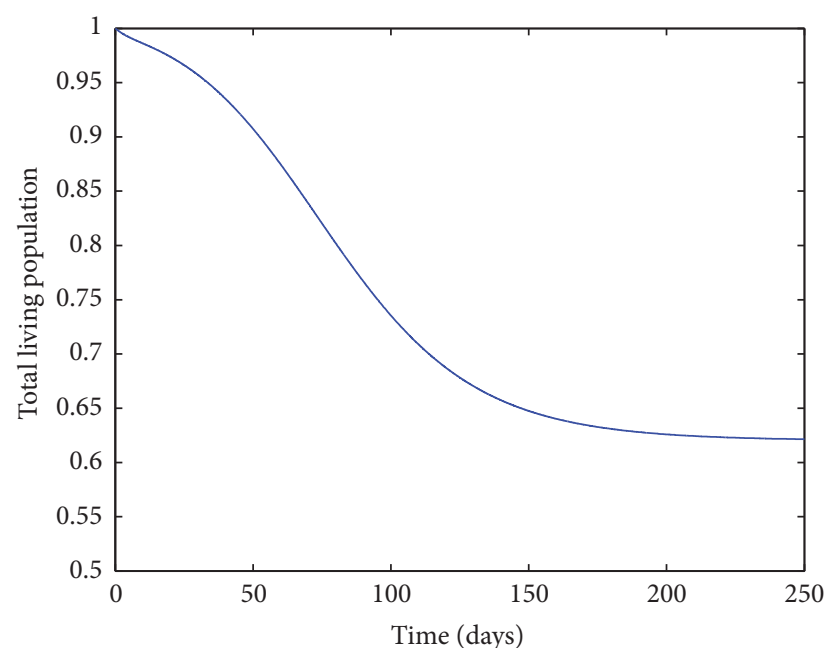

FIGURE 13: Evolution of the total alive population with daily culling rate of $\rho_{D}=0.1$ and constant vaccination of $V=V_{0}=0.2 b_{1}$.

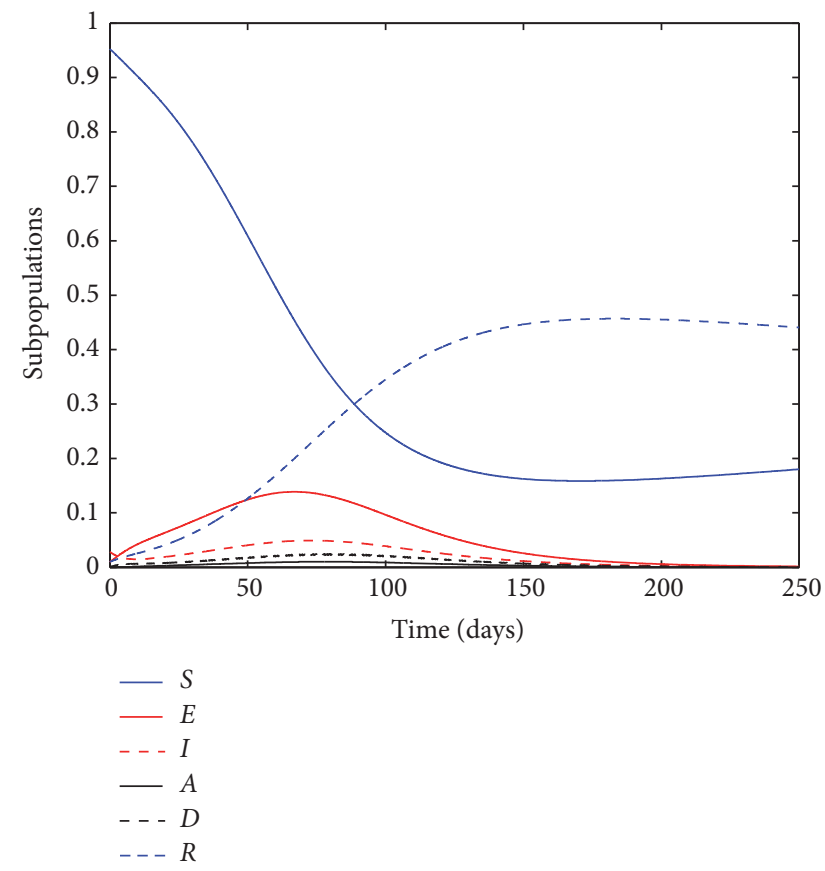

FIGURE 14: Evolution of the subpopulations with daily culling rate of $\rho_{D}=0.1$ and constant vaccination of $V=V_{0}=0.8 b_{1}$.

$0.01 I(t)$ to show its effect on the system. Figures 20 and 21 show the combined effect of the three external actions.

From Figures 17 and 21 it is observed that the total living population is improved thanks to the use of antivirals while the deaths associated with the disease are stopped due to the use of the proposed approach. Moreover, it is now worth comparing the behavior of the natural system without any kind of external action with the evolution of the system when culling, vaccination, and antivirals are applied, especially Figures 2 and 21. After 250 days of epidemics, the total living population without any external action is of $56.47 \%$ while it is of $98.74 \%$ when the proposed dedicated policies are applied. These values show the great success in the application of

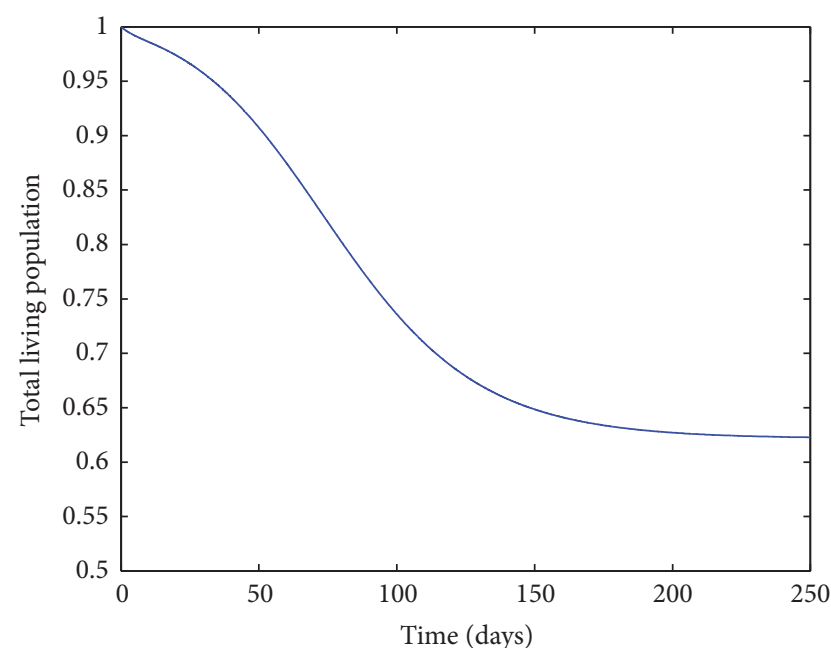

FIGURE 15: Evolution of the total alive population with daily culling rate of $\rho_{D}=0.1$ and constant vaccination of $V=V_{0}=0.8 b_{1}$.

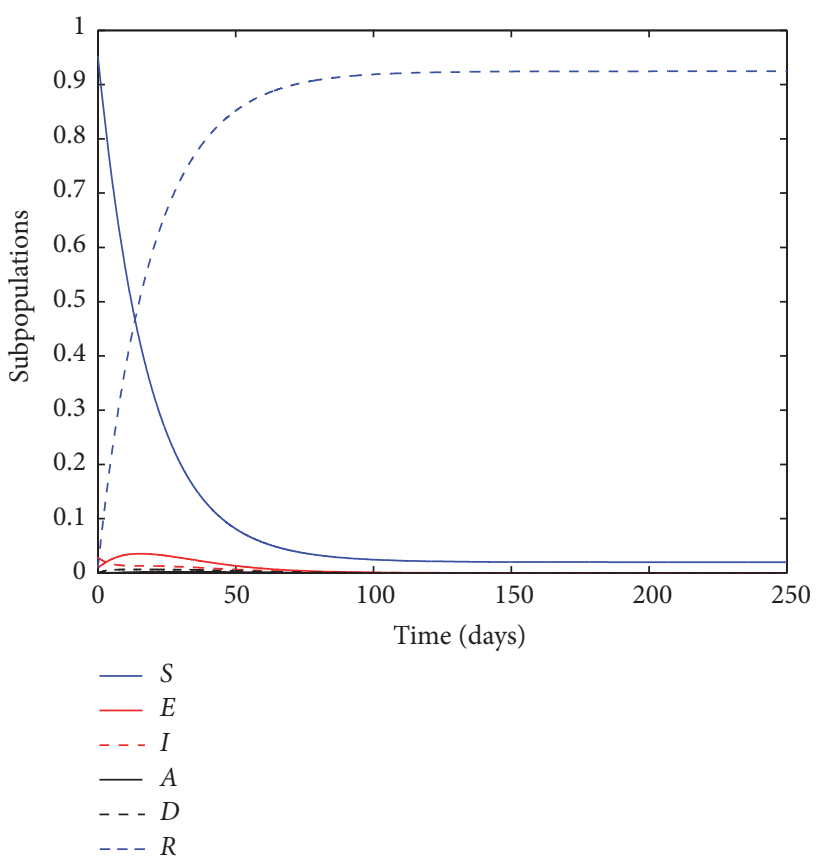

FIGURE 16: Evolution of the subpopulations with daily culling rate of $\rho_{D}=0.1$ and feedback vaccination of $V=0.2 b_{1}+0.002 S(t)$.

control measurements to lessen the impact of epidemics in society. Moreover, Figures 22, 23, and 24 show the control efforts associated with each one of the therapies. It is shown that the culling and antiviral actions vanish asymptotically so that they are only applied for a limited period of time while vaccination needs to be maintained since it converges to a positive constant.

Figures 25-28 show the behaviors of the asymptomatic and lying infective corpses under a culling rate of $\rho_{D}=0.1$. The oscillatory nature of the solution due to the impulsive culling action on infective corpses is better figured out in Figure 28 which is ran on longer observation time intervals. 


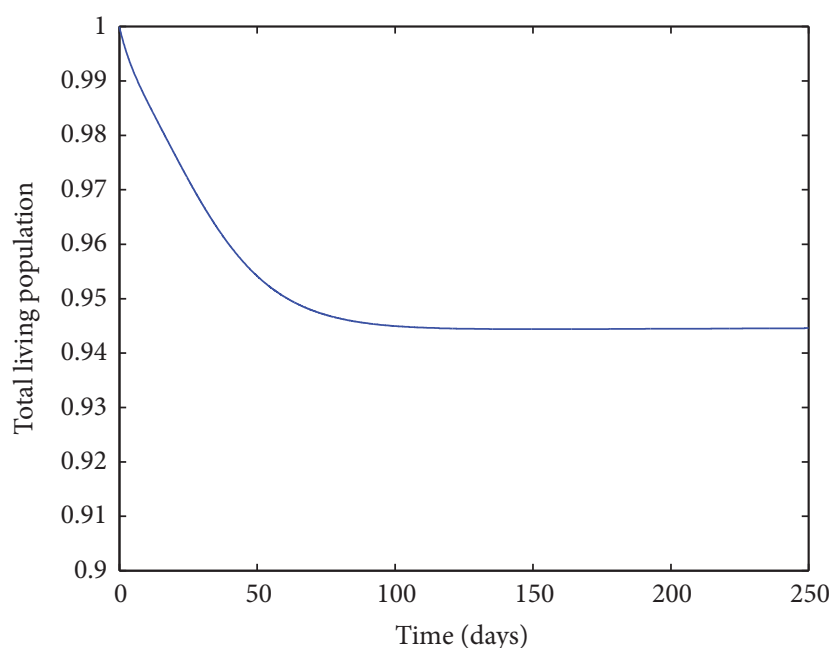

FIGURE 17: Evolution of the total alive population with daily culling rate of $\rho_{D}=0.1$ and feedback vaccination of $V=0.2 b_{1}+0.002 S(t)$.

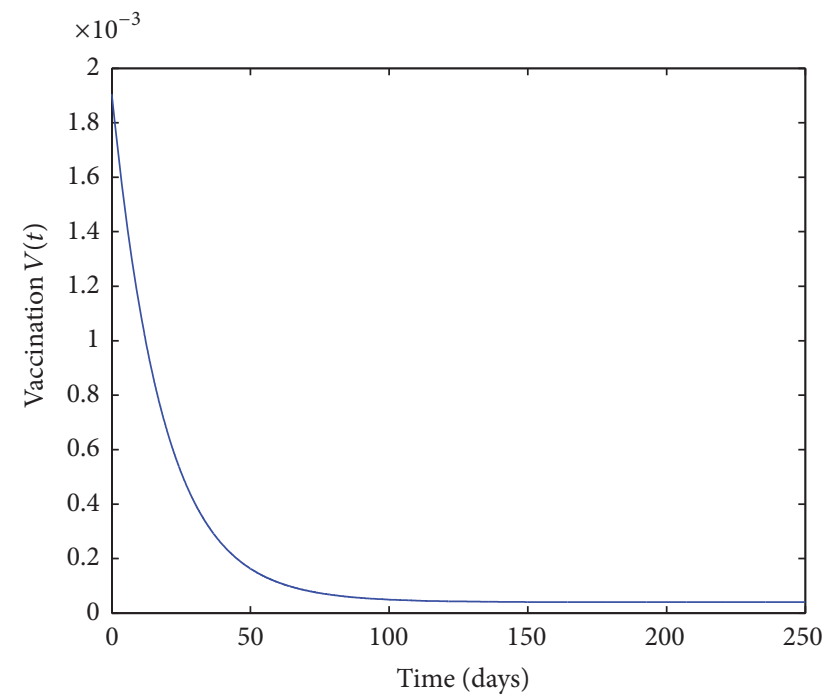

Figure 18: Vaccination function $V=0.2 b_{1}+0.002 S(t)$ when a daily culling rate of $\rho_{D}=0.1$ is also applied.

\section{Conclusions}

A new epidemic model is proposed with six subpopulations by incorporating the asymptomatic infectious and the dead corpses into a basic SEIR model of four subpopulations. The model is driven by three simultaneous controls in terms of a vaccination control on the susceptible which is based on linear time-varying feedback plus a constant term, an antiviral treatment on the symptomatic infectious subpopulation with infection feedback information, and a culling action of impulsive type on the infective dead corpses. The vaccination controls are combinations of feedback-independent (which can be constant, in particular) and feedback time-varying linear terms and the antiviral treatment control is of a timevarying linear feedback nature. There is also an impulsive time-dependent control action consisting of the retirement

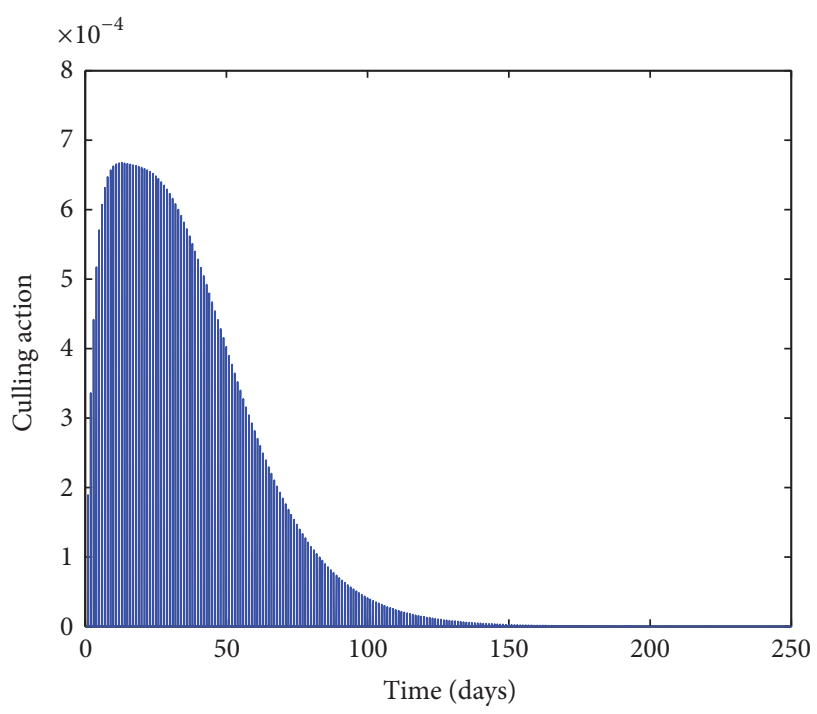

FIGURE 19: Culling effort $\rho_{D} D(t)$ when a daily culling rate of $\rho_{D}=0.1$ and vaccination law $V=0.2 b_{1}+0.002 S(t)$ are applied.

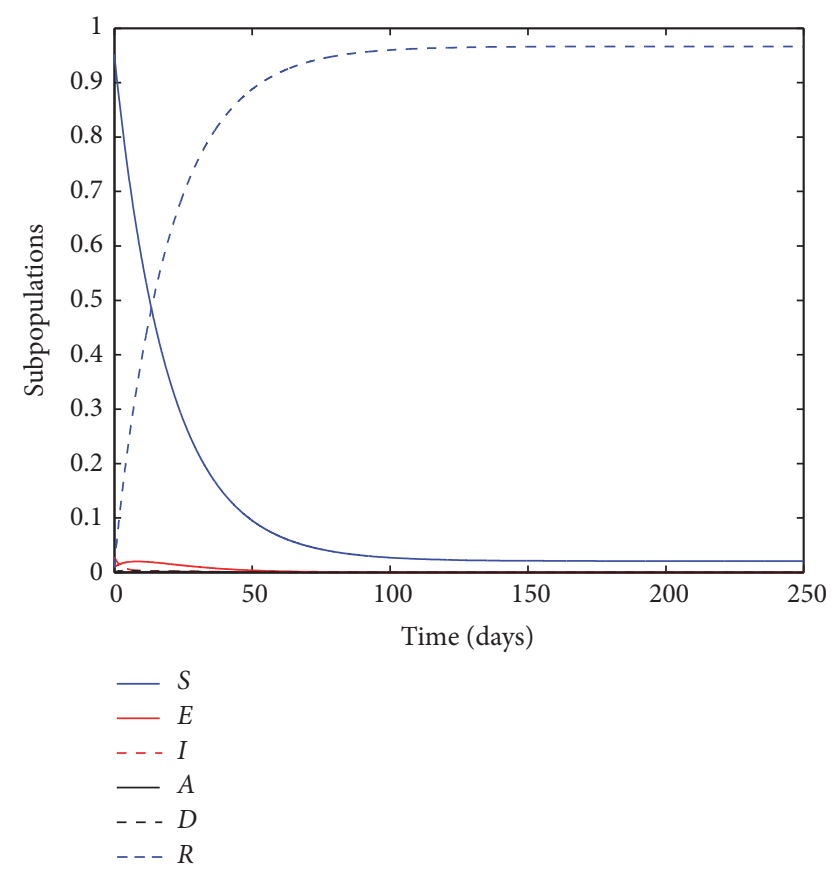

FIgURE 20: Evolution of the subpopulations with daily culling rate of $\rho_{D}=0.1$, feedback vaccination $V=0.2 b_{1}+0.002 S(t)$, and antiviral treatment $\xi(t)=K_{\xi} I(t)=0.01 I(t)$.

of corpses so as to reduce the risks of dead-contagion to the living uninfected population.

An identification and analysis of the endemic and diseasefree equilibrium points and equilibrium oscillations are performed in the case that the control gains are constant. The equilibrium oscillations arise as a generalization of the equilibrium points when the dead corpses recovery action has a periodic nature. The parameterizations of those mentioned steady-state solutions are investigated as being dependent on the control gains as they converge to constant values. The local stability properties of the steady states and the global 


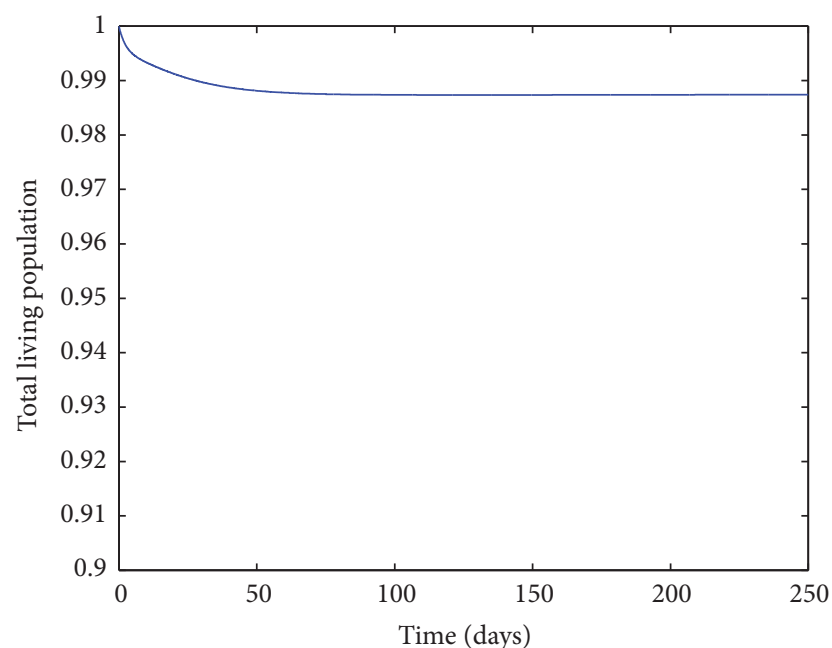

FIGURE 21: Evolution of the total alive population with daily culling rate of $\rho_{D}=0.1$, feedback vaccination $V=0.2 b_{1}+0.002 S(t)$, and antiviral treatment $\xi(t)=K_{\xi} I(t)=0.01 I(t)$.

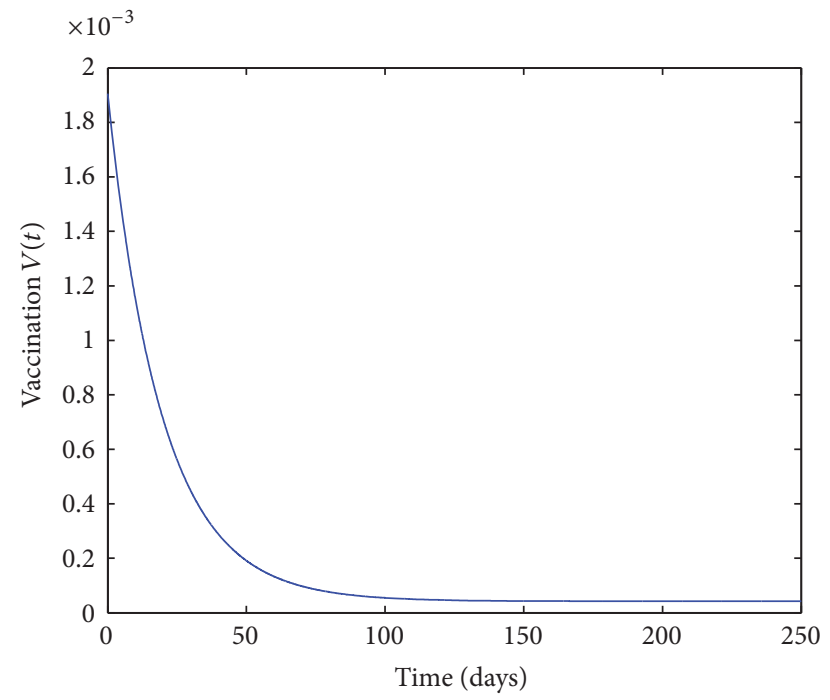

FIGURE 22: Vaccination function $V=0.2 b_{1}+0.002 S(t)$ when a daily culling rate of $\rho_{D}=0.1$ and antiviral treatment $\xi(t)=K_{\xi} I(t)=$ $0.01 I(t)$ are applied.

stability are investigated. The main novelties of the paper are (a) the incorporation of the asymptomatic infectious subpopulation and dead corpses as extra subpopulations with study of their steady states being either equilibrium points or oscillations; (b) the design of three distinct controls on the above proposed extended SEIADR model which can be time varying and with feedback information on the susceptible, symptomatic infections and dead corpses; (c) the performance of the global stability analysis based on qualitative theory of differential equations rather than on the analysis of Lyapunov functionals; and (d) the emphasis, supported within a variety of performed simulations, that the infection evolution might be very sensitive to the corpses culling action (impulsive control) parameters.

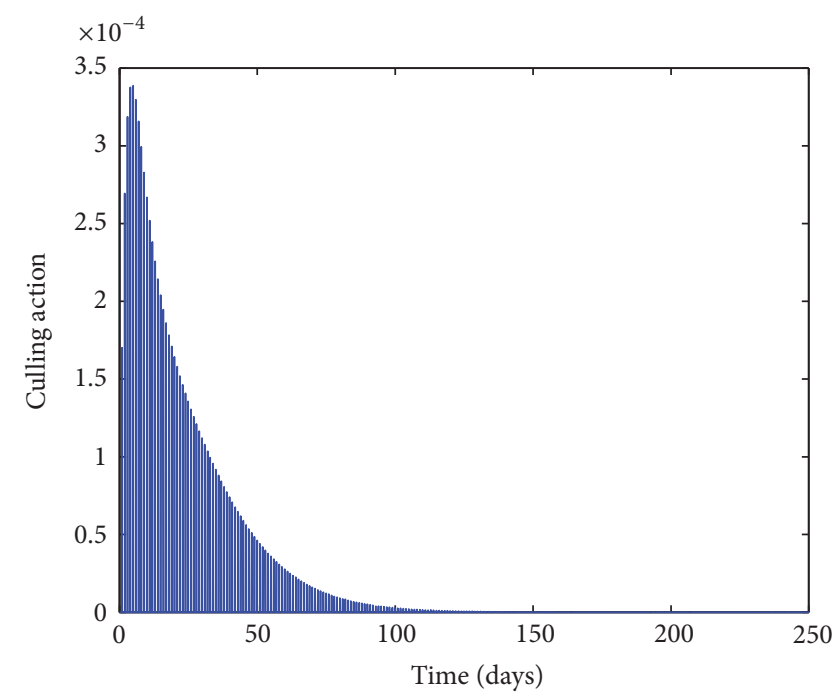

FIgURE 23: Culling effort $\rho_{D} D(t)$ when a daily culling rate of $\rho_{D}=$ 0.1 , vaccination law $V=0.2 b_{1}+0.002 S(t)$ and antiviral treatment $\xi(t)=K_{\xi} I(t)=0.01 I(t)$ are applied.

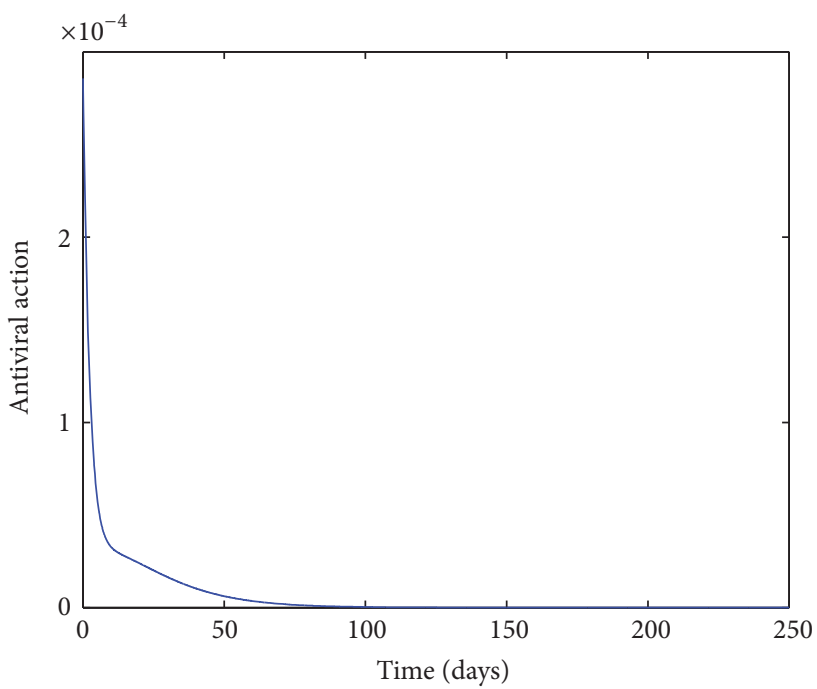

FIgURE 24: Antiviral action when a daily culling rate of $\rho_{D}=0.1$, vaccination law $V=0.2 b_{1}+0.002 S(t)$, and antiviral treatment $\xi(t)=$ $K_{\xi} I(t)=0.01 I(t)$ are applied.

\section{Appendix}

Proof of Theorem 10. Rewrite (2) equivalently as

$$
\begin{aligned}
\dot{E}(t) & -\left(\beta I(t)+\beta_{A} A(t)+\beta_{D} D(t)\right) S(t) \\
= & F_{1}(E(t), I(t)+A(t))=F_{1}(E(t), 0) \\
& :=-\left(b_{2}+\gamma\right) E(t)
\end{aligned}
$$




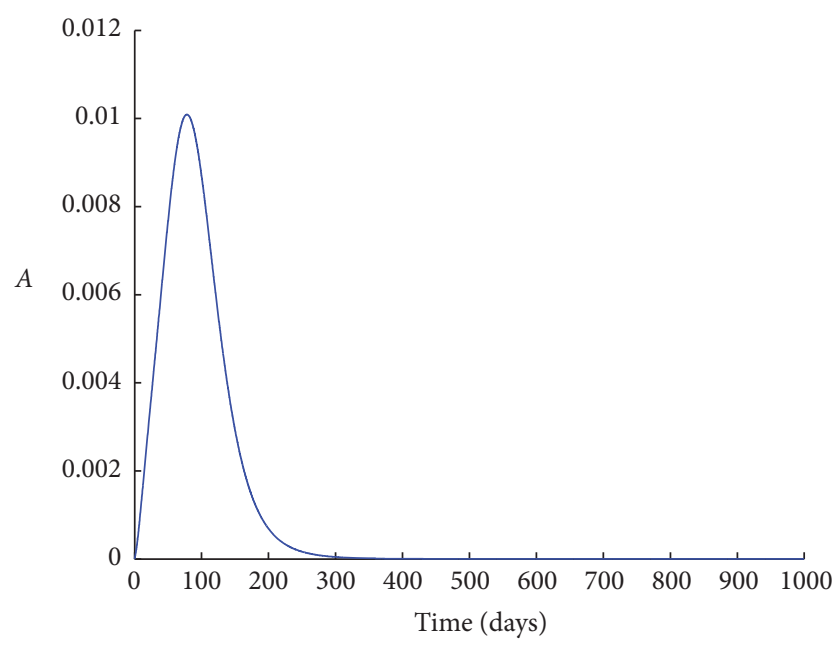

FIGURE 25: Evolution of the asymptomatic subpopulation with a daily culling rate of $\rho_{D}=0.1$. while one gets from (3), (4), and (8)

$$
\begin{aligned}
\dot{I}(t) & +\dot{A}(t)+\left(\alpha+K_{\xi}^{*}+\widetilde{K}_{\xi}(t)\right) I(t) \\
= & F_{2}(E(t), I(t)+A(t)) \\
& :=\gamma E(t)-\left(b_{2}+\tau_{0}\right)(I(t)+A(t)),
\end{aligned}
$$

where $\widetilde{K}_{\xi}(t)=K_{\xi}(t)-K_{\xi}^{*}$. Note from (A.1)-(A.2) that $F_{1}(E(t), 0)$ and $F_{2}(E(t), I(t)+A(t))$ are continuous with continuous partial derivatives with respect to their arguments in any simply connected region $\mathbf{C}_{\text {int }}$ of $\mathbf{R}^{2}$

$$
\begin{gathered}
\frac{\partial F_{1}(E(t), 0)}{\partial E(t)}+\frac{\partial F_{2}(E(t), I(t)+A(t))}{\partial(I(t)+A(t))} \\
=-\left(2 b_{2}+\gamma+\tau_{0}\right)<0 ; \quad \forall t \in \mathbf{R}_{0+} .
\end{gathered}
$$

Any such region $\mathbf{C}_{\text {int }}$ cannot contain a closed trajectory $\mathbf{C}$ (limit cycle) from Gauss-Stokes theorem since then

$$
\begin{aligned}
& \oint_{\mathrm{C}} {\left[F_{1}(E(t), 0) d(I(t)+A(t))-F_{2}(E(t), I(t)+A(t)) d E(t)\right] } \\
&=\iint_{\mathrm{C}_{\text {int }}}\left(\frac{\partial F_{1}(E(t), 0)}{\partial E(t)}+\frac{\partial F_{2}(E(t), I(t)+A(t))}{\partial(I(t)+A(t))}\right) d E(t) d(I(t)+A(t))<0 ;
\end{aligned}
$$

from (A.3) if $\mathbf{C}_{\text {int }_{C}}$ is the interior of the set defined by the simple curve C, a contradiction, (Bendixson's criterion of nonexistence of limit cycles [34] or Bendixson's first theorem, implies that the above integral has to be null for closed trajectories) then it should hold $\dot{\bar{F}}_{2}(t) d \bar{F}_{1}(t)-\dot{\bar{F}}_{1}(t) d \bar{F}_{2}(t)=0$ along the orbit $\mathbf{C}$ and this is impossible from (A.4), where

$$
\begin{aligned}
\bar{F}_{1}(t) & \\
= & E(t)-E(0) \\
& \quad-\int_{0}^{t}\left(\beta I(\sigma)+\beta_{A} A(\sigma)+\beta_{D} D(\sigma)\right) S(\sigma) d \sigma \\
= & -\left(b_{2}+\gamma\right) \int_{0}^{t} E(\sigma) d \sigma \\
\bar{F}_{2}(t) & \\
= & I(t)+A(t)-I(0)-A(0) \\
& +\int_{0}^{t}\left(\alpha+K_{\xi}^{*}+\widetilde{K}_{\xi}(\sigma)\right) I(\sigma) d \sigma \\
= & \int_{0}^{t}\left(\gamma E(\sigma)-\left(b_{2}+\tau_{0}\right)(I(\sigma)+A(\sigma))\right) d \sigma
\end{aligned}
$$

from (A.1)-(A.2). Since $\widetilde{K}_{\xi}(t) \rightarrow 0$ as $t \rightarrow \infty$ one has from (A.1) and (A.2) and (A.4) that

$$
\begin{gathered}
\lim _{t \rightarrow \infty}\left[\dot{I}(t)+\dot{A}(t)+\left(\alpha+K_{\xi}^{*}\right) I(t)\right. \\
\left.-F_{2}(E(t), I(t)+A(t))\right]=0,
\end{gathered}
$$

$$
\begin{aligned}
\lim _{t \rightarrow \infty} & {\left[\dot{E}(t)-\left(\beta I(t)+\beta_{A} A(t)+\beta_{D} D(t)\right) S(t)\right.} \\
- & \left.F_{1}(E(t), 0)\right]=0 .
\end{aligned}
$$

Taking Laplace transforms in (A.6) by neglecting initial conditions and using (48), one gets from (A.6) that $\widehat{E}(s)=$ $\widehat{F}_{2}(s) /\left(\left(C_{I}+C_{A}\right) s+\left(\alpha+K_{\xi}^{*}\right) C_{I}\right)$, where the superscript "hat" denotes the Laplace transform in the Laplace argument "s" of $F_{2}(\cdot)$. Since $F_{2}(t)$ is not asymptotically periodic the Laplace antitransform of $\widehat{E}(s)$, that is, $E(t)$, is not asymptotically periodic from the above expression. Since $E(t)$ is not asymptotically periodic then $I(t)$ and $A(t)$ and $D(t)$ are not asymptotically periodic (note the assumption $\rho_{D}^{*}=0$ ). On the other hand, one gets from (6) to (8) as $t \rightarrow \infty$, since $K_{V}(t) \rightarrow K_{V}^{*}$ and $K_{\xi}(t) \rightarrow K_{\xi}^{*}$ as $t \rightarrow \infty$ that

$$
\begin{gathered}
\dot{R}(t)+\left(b_{2}+\eta\right) R(t)-V_{0}-K_{V}^{*} S(t) \\
=\tau_{0} A(t)+\left(\tau_{0}+K_{\xi}^{*}\right) I(t)
\end{gathered}
$$

while summing up (1) and (6) by taking into account (2) and (48) yields

$$
\begin{aligned}
\dot{S}(t) & +\dot{R}(t)+b_{2}(S(t)+R(t)) \\
= & -\dot{E}(t)+b_{1} \\
& +\left(\tau_{0} C_{A}+\left(\tau_{0}+K_{\xi}^{*}\right) C_{I}-\left(b_{2}+\gamma\right)\right) E(t) .
\end{aligned}
$$




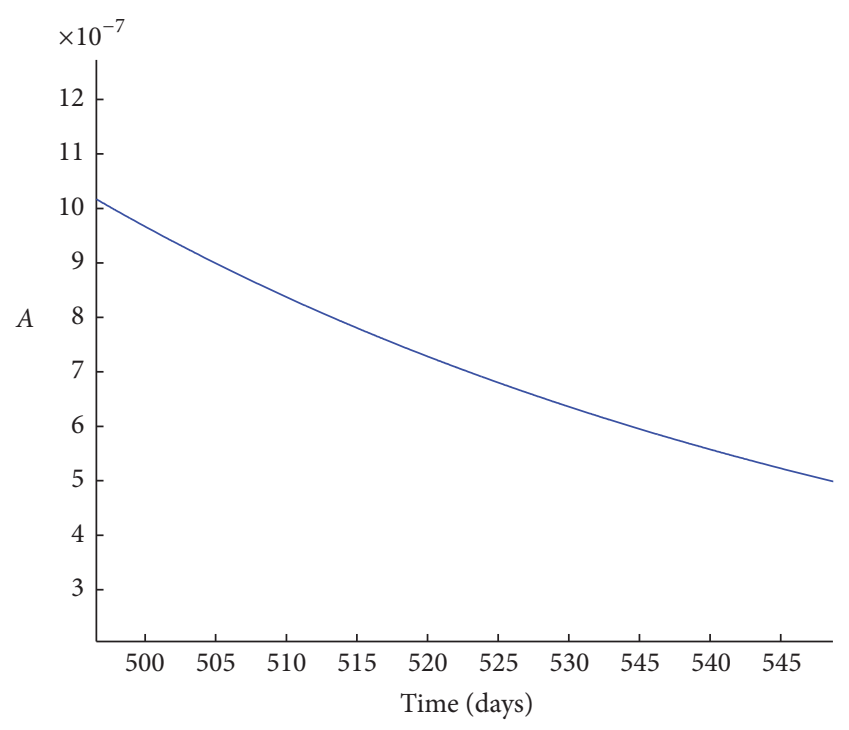

FIGURE 26: Zoom on the evolution of the asymptomatic subpopulation with a daily culling rate of $\rho_{D}=0.1$.

Subtracting (A.8) from (A.9) and rewriting (A.9) in an equivalent form yields

$$
\begin{aligned}
& \dot{S}(t)+\dot{E}(t)+\left(b_{2}+\gamma\right) E(t)-b_{1}+V_{0} \\
& =F_{3}(S(t), R(t)):=-\left(b_{2}+K_{V}^{*}\right) S(t)+\eta R(t), \\
& \dot{S}(t)+\dot{R}(t)+\dot{E}(t)-b_{1} \\
& \quad+\left(b_{2}+\gamma-\tau_{0} C_{A}-\left(\tau_{0}+K_{\xi}^{*}\right) C_{I}\right) E(t) \\
& =F_{4}(S(t), R(t)):=-b_{2}(S(t)+R(t)) \\
& \frac{\partial F_{3}(S(t), R(t))}{\partial S(t)}+\frac{\partial F_{4}(S(t), R(t))}{\partial R(t)} \\
& =-\left(2 b_{2}+K_{V}^{*}\right)<0
\end{aligned}
$$

$$
\forall t \in \mathbf{R}_{0+} \text {. }
$$

Since $\operatorname{sign}\left(\left(\partial F_{3}(S(t), R(t))\right) / \partial S(t)+\left(\partial F_{4}(S(t), R(t))\right) / \partial R(t)\right)$ is constant along state-trajectory solutions in $\mathbf{R}^{2}$, one has again that no closed trajectory (then no limit cycle) can exist surrounding any region with Poincaré's index +1 . In view of (A.11)-(A.12), the functions $\dot{S}(t)+\dot{E}(t)+\left(b_{2}+\gamma\right) E(t)-b_{1}+V_{0}$ and $\dot{S}(t)+\dot{R}(t)+\dot{E}(t)-b_{1}+\left(b_{2}+\gamma-\tau_{0} C_{A}-\left(\tau_{0}+K_{\xi}^{*}\right) C_{I}\right) E(t)$ are not asymptotically periodic. Since $E(t)$ and $\dot{E}(t)$ have been proved to be nonasymptotically periodic then $(\dot{S}(t)+\dot{R}(t)), \dot{S}(t), \dot{R}(t)$, and then their time-integral solutions are not asymptotically periodic either.

The above arguments, together with the property of uniform boundedness of the total population and that of the nonnegativity of the solution, conclude that if only the disease-free equilibrium point exists while it is locally asymptotically stable then it is globally asymptotically stable as well since no limit cycle can exist around it in any plane in $\mathbf{R}_{0+}^{2}$ associated with any two of the state variables. On the other hand, assume that the endemic equilibrium state

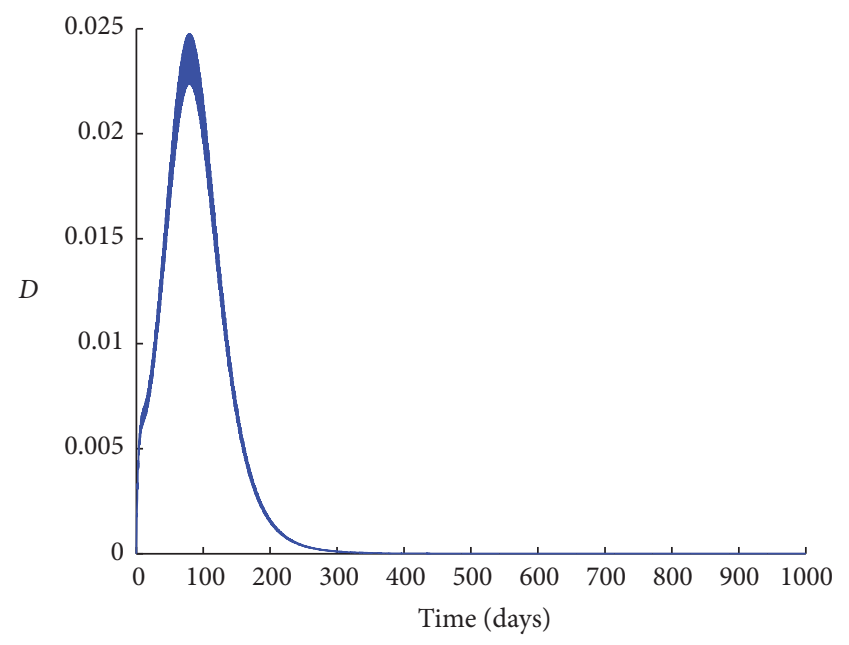

FIgURE 27: Evolution of the infective lying corpses with a daily culling rate of $\rho_{D}=0.1$.

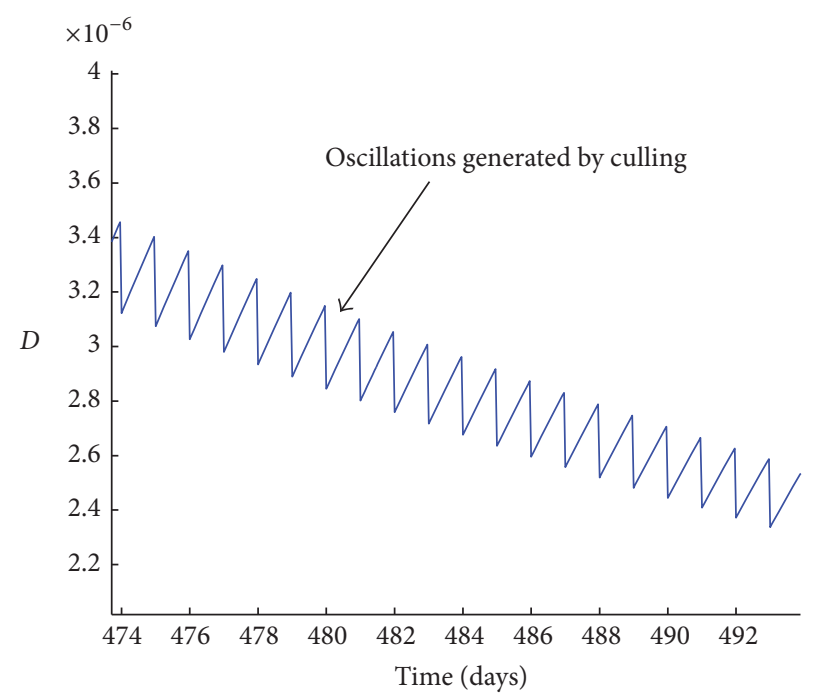

FIGURE 28: Zoom on the evolution of the infective corpses with a daily culling rate of $\rho_{D}=0.1$.

is not a stable attractor while the disease-free is unstable. Then, an unstable limit cycle around it cannot exist from the above discussion (which excludes both stable and instable limit cycles) and, due to the nonnegativity of the solution and to the uniform boundedness of the whole population, then the trajectory converges asymptotically to it so that it is a stable attractor.

If the disease-free equilibrium point is unstable and the endemic equilibrium exists then the endemic equilibrium point is a stable attractor and the system is globally asymptotically stable with any state-trajectory solution converging to it.

Two other possible stability/instability combinations of the stability of both equilibrium states are excluded as follows leading to Property (ii):

(1) The case that both equilibrium states are simultaneously locally stable is excluded. Since there is no 
closed trajectory solution then there is no semistable limit cycle separating the domains of attraction of both equilibrium states within the first orthant of $\mathbf{R}^{6}$.

(2) The case that both equilibrium states are simultaneously unstable is excluded as well since the system is globally stable if it is positive.

\section{Competing Interests}

The authors declare that they do not have any competing interests.

\section{Authors' Contributions}

All the authors contributed equally to all the parts of the manuscript.

\section{Acknowledgments}

This research is supported by the Spanish Government and the European Fund of Regional Development FEDER through Grant DPI2015-64766-R.

\section{References}

[1] D. Mollison, Ed., Epidemic Models: Their Structure and Relation to Data, Publications of the Newton Institute, Cambridge University Press, 1995, (transferred to digital printing 2003).

[2] M. J. Keeling and P. Rohani, Modeling Infectious Diseases in Humans and Animals, Princeton University Press, Princeton, NJ, USA, 2008.

[3] D. J. Daley and J. Gani, Epidemic Modelling. An Introduction, vol. 15 of Cambridge Studies in Mathematical Biology, Cambridge University Press, New York, NY, USA, 2005.

[4] H. Khan, R. N. Mohapatra, K. Vajravelu, and S. J. Liao, "The explicit series solution of SIR and SIS epidemic models," Applied Mathematics and Computation, vol. 215, no. 2, pp. 653-669, 2009.

[5] X. Song, Y. Jiang, and H. Wei, "Analysis of a saturation incidence SVEIRS epidemic model with pulse and two time delays," Applied Mathematics and Computation, vol. 214, no. 2, pp. 381390, 2009.

[6] M. De la Sen, R. P. Agarwal, A. Ibeas, and S. Alonso-Quesada, "On the existence of equilibrium points, boundedness, oscillating behavior and positivity of a SVEIRS epidemic model under constant and impulsive vaccination," Advances in Difference Equations, vol. 2011, Article ID 748608, 2011.

[7] M. De la Sen, R. P. Agarwal, A. Ibeas, and S. Alonso-Quesada, "On a generalized time-varying SEIR epidemic model with mixed point and distributed time-varying delays and combined regular and impulsive vaccination," Advances in Difference Equations, vol. 2010, Article ID 281612, 2010.

[8] M. De la Sen and S. Alonso-Quesada, "Vaccination strategies based on feedback control techniques for a general SEIRepidemic model," Applied Mathematics and Computation, vol. 218, no. 7, pp. 3888-3904, 2011.
[9] M. de la Sen, S. Alonso-Quesada, and A. Ibeas, "On the stability of an SEIR epidemic model with distributed timedelay and a general class of feedback vaccination rules," Applied Mathematics and Computation, vol. 270, pp. 953-976, 2015.

[10] Z. Fitriah and A. Suryanto, "Nonstandard finite difference scheme for SIRS epidemic model with disease- related death," in Proceedings of the Symposium on Biomathematics (SYMOMATH '15), vol. 1723 of AIP Conference Proceedings, Bandung, Indonesia, 2015.

[11] J. P. Tripathi and S. Abbas, "Global dynamics of autonomous and nonautonomous SI epidemic models with nonlinear incidence rate and feedback controls," Nonlinear Dynamics, vol. 86, no. 1, pp. 337-351, 2016.

[12] Z. Wei and M. Le, "Existence and convergence of the positive solutions of a discrete epidemic model," Discrete Dynamics in Nature and Society, vol. 2015, Article ID 434537, 10 pages, 2015.

[13] X. Wang, "An SIRS epidemic model with vital dynamics and a ratio-dependent saturation incidence rate," Discrete Dynamics in Nature and Society. An International Multidisciplinary Research and Review Journal, vol. 2015, Article ID 720682, 9 pages, 2015.

[14] L. Wang, Z. Liu, and X. Zhang, "Global dynamics of an SVEIR epidemic model with distributed delay and nonlinear incidence," Applied Mathematics and Computation, vol. 284, pp. 47-65, 2016.

[15] F. Wei and F. Chen, "Stochastic permanence of an SIQS epidemic model with saturated incidence and independent random perturbations," Physica A: Statistical Mechanics and Its Applications, vol. 453, pp. 99-107, 2016.

[16] L. Shaikhet and A. Korobeinikov, "Stability of a stochastic model for HIV-1 dynamics within a host," Applicable Analysis, vol. 95, no. 6, pp. 1228-1238, 2016.

[17] L. Shaikhet, "Stability of equilibrium states for a stochastically perturbed exponential type system of difference equations," Journal of Computational and Applied Mathematics, vol. 290, pp. 92-103, 2015.

[18] R. Peng and F. Yi, "Asymptotic profile of the positive steady state for an SIS epidemic reaction-diffusion model: effects of epidemic risk and population movement," Physica D. Nonlinear Phenomena, vol. 259, pp. 8-25, 2013.

[19] R. Peng, "Asymptotic profiles of the positive steady state for an SIS epidemic reaction-diffusion model. Part I," Journal of Differential Equations, vol. 247, no. 4, pp. 1096-1119, 2009.

[20] B. Buonomo, D. Lacitignola, and C. Vargas-De-Leon, "Qualitative analysis and optimal control of an epidemic model with vaccination and treatment," Mathematics and Computers in Simulation, vol. 100, pp. 88-102, 2014.

[21] K. Mcculloch, M. G. Roberts, and C. R. Laing, "Exact analytical expressions for the final epidemic size of an SIR model on small networks," The ANZIAM Journal, vol. 57, no. 4, pp. 429-444, 2016.

[22] L. Ling, G. Jiang, and T. Long, "The dynamics of an SIS epidemic model with fixed-time birth pulses and state feedback pulse treatments," Applied Mathematical Modelling, vol. 39, no. 18, pp. 5579-5591, 2015.

[23] Y. He, S. Gao, and D. Xie, "An SIR epidemic model with timevarying pulse control schemes and saturated infectious force," Applied Mathematical Modelling, vol. 37, no. 16-17, pp. 8131-8140, 2013.

[24] S. Sharma and G. P. Samanta, "Stability analysis and optimal control of an epidemic model with vaccination," International 
Journal of Biomathematics, vol. 8, no. 3, Article ID 1550030, 28 pages, 2015.

[25] G. P. Samanta, "A delayed hand-foot-mouth disease model with pulse vaccination strategy," Computational and Applied Mathematics, vol. 34, no. 3, pp. 1131-1152, 2015.

[26] M. R. de Pinho, I. Kornienko, and H. Maurer, "Optimal control of a SEIR model with mixed constraints and $L^{1}$ cost," in CONTROLO '2014-Proceedings of the 11th Portuguese Conference on Automatic Control, vol. 321 of Lecture Notes in Electrical Engineering, Springer, Basel, Switzerland, 2015.

[27] E. Santermans, E. Robesyn, T. Ganyani et al., "Spatiotemporal evolution of Ebola virus disease at sub-national level during the 2014 West Africa epidemic: model scrutiny and data meagreness," PLoS ONE, vol. 11, no. 1, Article ID e0147172, 2016.

[28] I. Al-Darabsah and Y. Yuan, "A time-delayed epidemic model for Ebola disease transmission," Applied Mathematics and Computation, vol. 290, pp. 307-325, 2016.

[29] S. E. Bellan, J. R. C. Pulliam, J. Dushoff, and L. A. Meyers, "Ebola control: effect of asymptomatic infection and acquired immunity," The Lancet, vol. 384, no. 9953, pp. 1499-1500, 2014.

[30] M. de la Sen, "Preserving positive realness through discretization," Positivity, vol. 6, no. 1, pp. 31-45, 2002.

[31] B. Xie, Z. Wang, Y. Xue, and Z. Zhang, "The dynamics of a delayed predator-prey model with double Allee effect," Discrete Dynamics in Nature and Society, vol. 2015, Article ID 102597, 8 pages, 2015.

[32] X. Wen, Y. Chen, and H. Yin, "Positive solutions of a diffusive predator-prey system including disease for prey and equipped with Dirichlet boundary condition," Discrete Dynamics in Nature and Society, vol. 2016, Article ID 2323752, 10 pages, 2016.

[33] J. M. Ortega, Numerical Analysis, Academic Press, New York, NY, USA, 1972.

[34] J. E. Gibson, Nonlinear Automatic Control, McGraw-Hill, New York, NY, USA, 1963. 


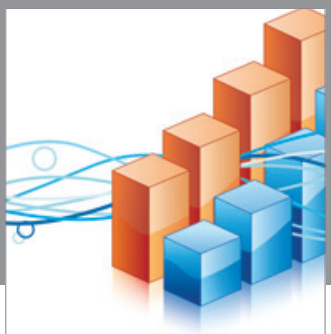

Advances in

Operations Research

vatem alat4

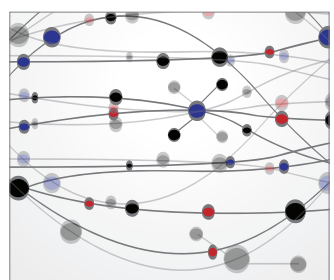

\section{The Scientific} World Journal
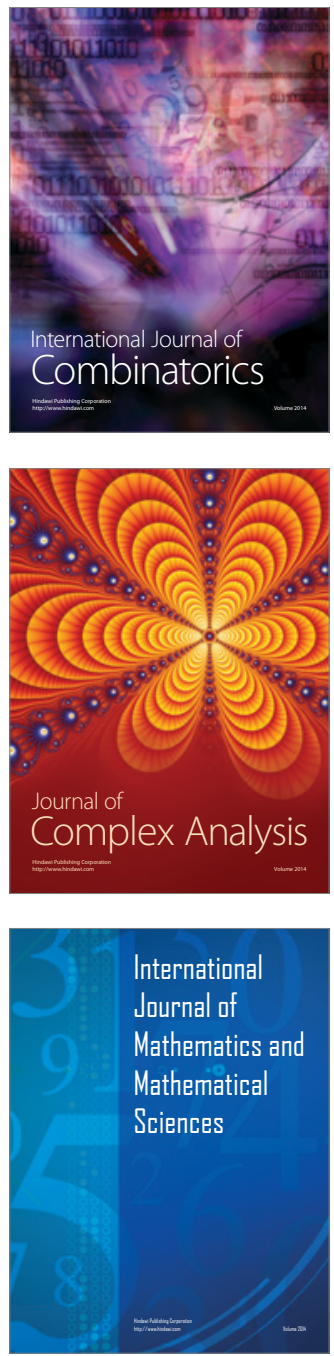
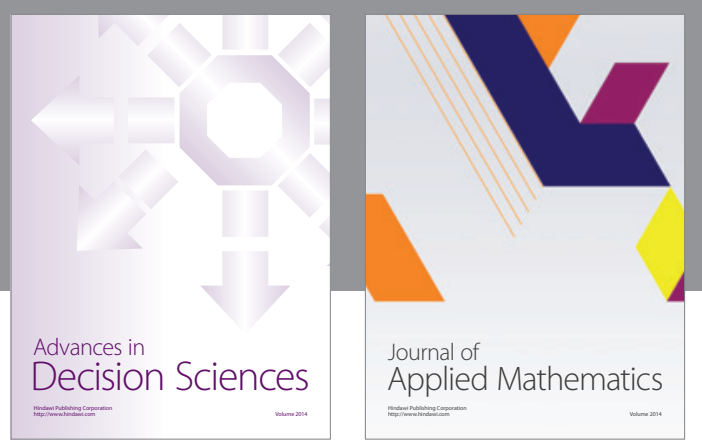

Algebra

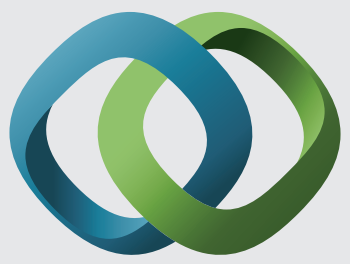

\section{Hindawi}

Submit your manuscripts at

https://www.hindawi.com
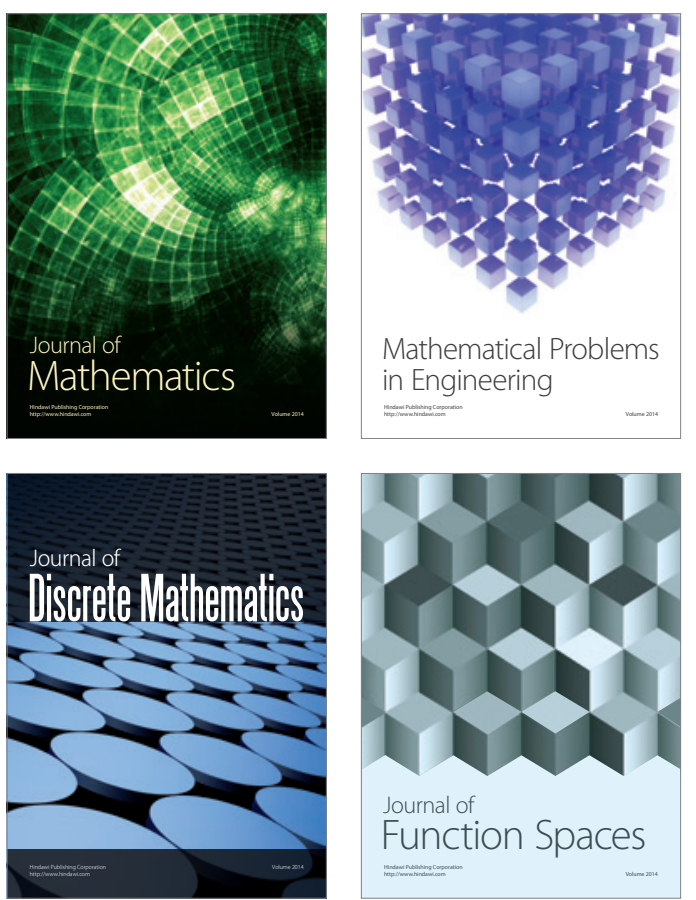

Mathematical Problems in Engineering
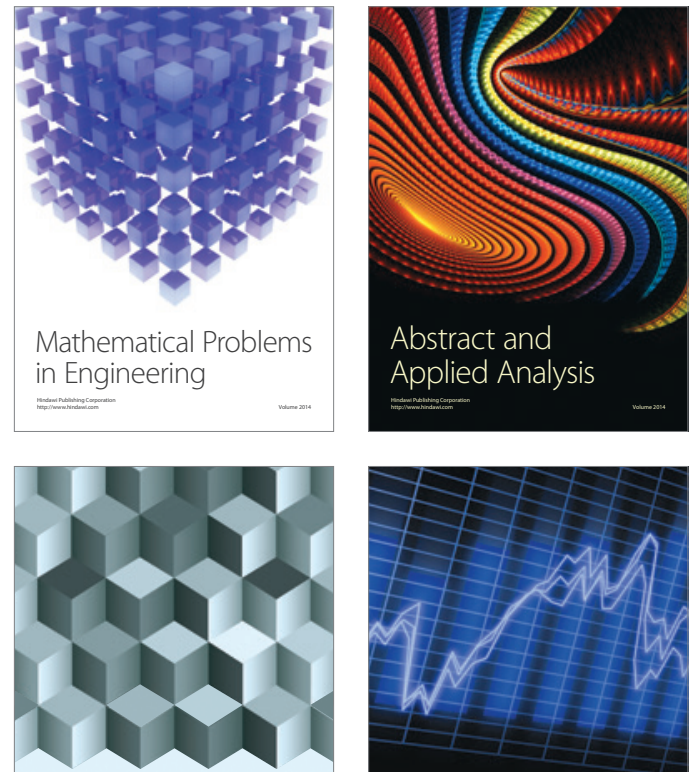

Journal of

Function Spaces

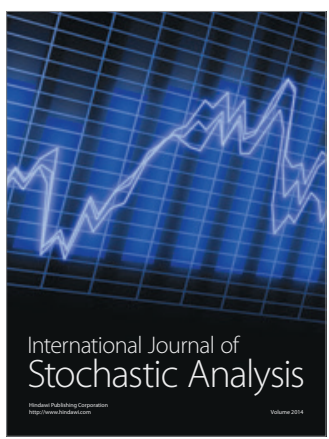

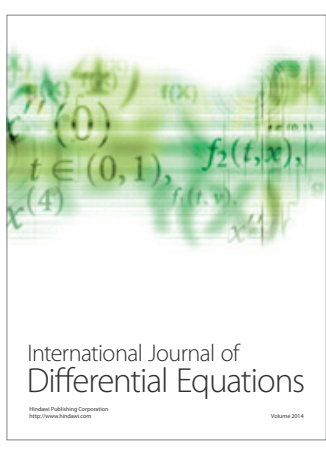
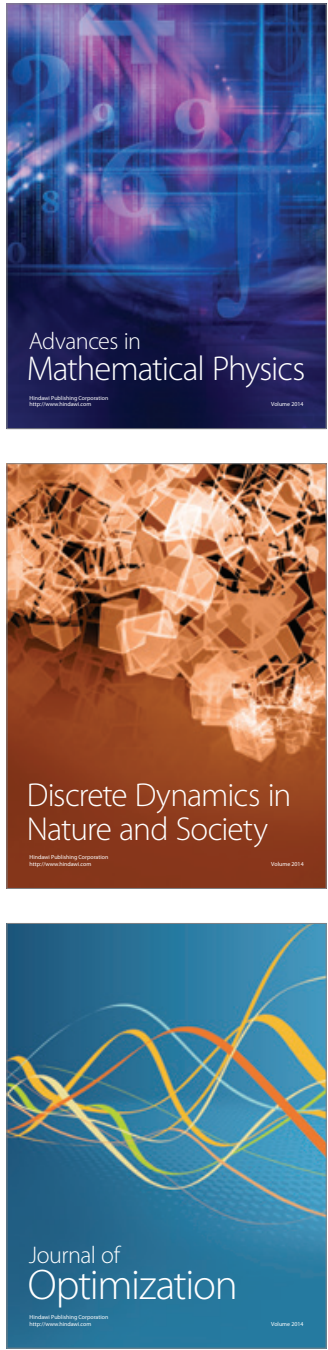